Florida International University

FIU Digital Commons

6-28-2019

\title{
The Neural Recruitment of Executive Function in Monolingual versus Bilingual Preterm-born Children: An FNIRS Study
}

Caitlyn F. Myland

Florida International University, cmyla001@fiu.edu

Follow this and additional works at: https://digitalcommons.fiu.edu/etd

Part of the Biomedical Engineering and Bioengineering Commons

\section{Recommended Citation}

Myland, Caitlyn F., "The Neural Recruitment of Executive Function in Monolingual versus Bilingual Pretermborn Children: An FNIRS Study" (2019). FIU Electronic Theses and Dissertations. 4216.

https://digitalcommons.fiu.edu/etd/4216

This work is brought to you for free and open access by the University Graduate School at FIU Digital Commons. It has been accepted for inclusion in FIU Electronic Theses and Dissertations by an authorized administrator of FIU Digital Commons. For more information, please contact dcc@fiu.edu. 


\section{FLORIDA INTERNATIONAL UNIVERSITY}

Miami, Florida

\section{THE NEURAL RECRUITMENT OF EXECUTIVE FUNCTION IN MONOLINGUAL} VERSUS BILINGUAL PRETERM-BORN CHLDREN: AN FNIRS STUDY

A thesis submitted in partial fulfillment of

the requirements for the degree of

MASTER OF SCIENCE

in

BIOMEDICAL ENGINEERING

by

Caitlyn F. Myland 
To: Dean John L. Volakis

College of Engineering and Computing

This thesis, written by Caitlyn F. Myland, and entitled The Neural Recruitment of ExecutivE Function in Monolingual Versus Bilingual Preterm-Born Children: An fNIRS Study having been approved in respect to style and intellectual content, is referred to you for judgment.

We have read this thesis and recommend that it be approved.

\begin{tabular}{r}
\hline Wei-Chiang Lin \\
\hline Melissa Baralt \\
Ranu Jung, Major Professor
\end{tabular}

Date of Defense: June 28, 2019

The thesis of Caitlyn F. Myland is approved.

Dean John L. Volakis College of Engineering and Computing

Andrés G. Gil Vice President for Research and Economic Development and Dean of the University Graduate School

Florida International University, 2019 
(C) Copyright 2019 by Caitlyn F. Myland

All rights reserved. 


\section{DEDICATION}

I would like to dedicate this thesis to all preterm born children, families, and their physicians and nurses that they may be informed of all the possibilities that bilingualism can bring to their lives. 


\section{ACKNOWLEDGMENTS}

I would like to acknowledge my mentor Dr. Baralt, for all her love, guidance, and encouragement throughout this process. Whenever I would lose hope, she would effortlessly restore it, without even knowing. I would also like to acknowledge my advisor, Dr. Jung for advising me and helping me overcome obstacles that I faced to get me to this last round. I would like to also thank my third committee member, Dr. Lin, in addition to the fNIRS Team for all their hard work and diligence towards this study. Finally, I would like to acknowledge my mom, dad, siblings, Shade, Megan, Trish, and friends for helping me become the woman I am today and supporting me throughout my life and more importantly grad school. I could not have done it without you all, so thank you. 


\begin{abstract}
OF THE THESIS
THE NEURAL RECRUITMENT OF EXECUTIVE FUNCTION IN MONOLINGUAL VERSUS BILINGUAL PRETERM-BORN CHLDREN: AN FNIRS STUDY
\end{abstract}

by

Caitlyn F. Myland

Florida International University, 2019

Miami, Florida

Professor Ranu Jung, Major Professor

Children born preterm are at risk for deficiencies in executive functioning (EF) as compared to term-born children (Nosarti, Giouroukou, Micali, Rifkin, Morris, and Murray, 2007; Taylor \& Clark, 2016). While bilingualism has been shown to enhance the EF of term-born children (e.g., Bialystok \& Martin, 2004; Head, Baralt, \& Darcy Mahoney, 2015), it has yet to be explored whether bilingualism also enhances the EF of preterm-born children. The present study fills this gap by examining the EF of bilingual versus monolingual preterm-born children. Participants were 9 bilingual and 7 monolingual righthanded children, ages six to seven, and born before 35 weeks. Results showed that both groups performed about the same in accuracy on the Dimensional Change Card Sort (DCCS) and the Go/No-Go task, however, the bilingual children were significantly faster. fNIRS analysis revealed significantly different neural recruitment of EF: bilingual children required significantly less oxygenated hemoglobin, implying that less cognitive effort was necessary. 


\section{TABLE OF CONTENTS}

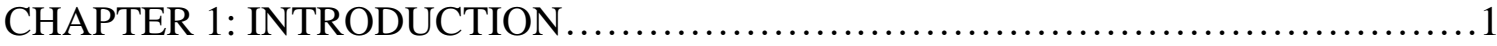

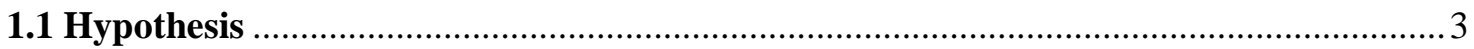

1.2 Rationale

1.3 Significance

CHAPTER 2: LITERATURE REVIEW .......................................6

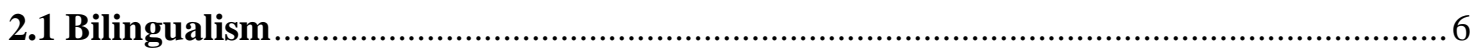

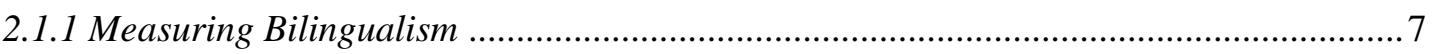

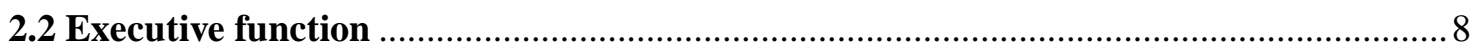

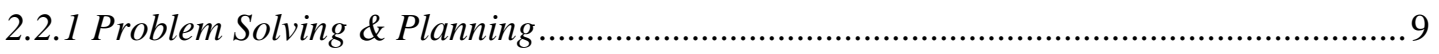

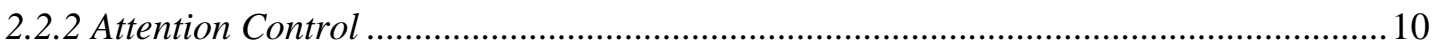

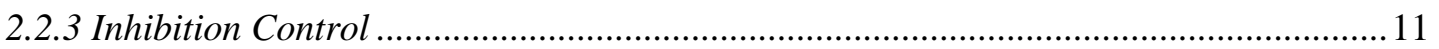

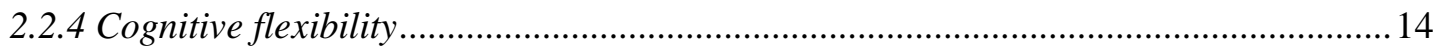

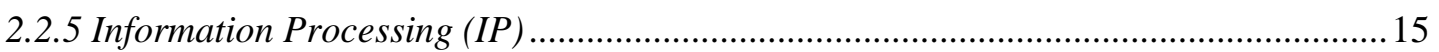

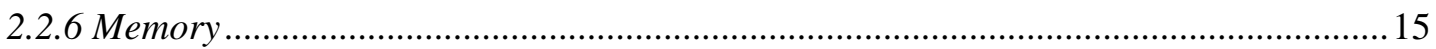

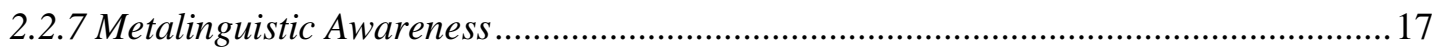

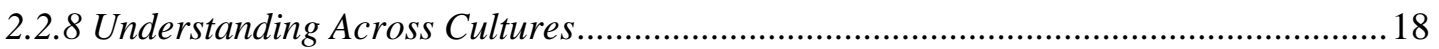

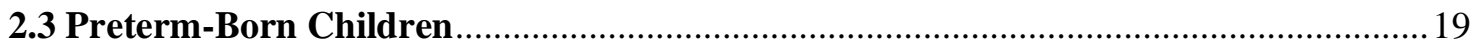

2.3.1 Effects of Prematurity on Executive Function ...................................................................2.

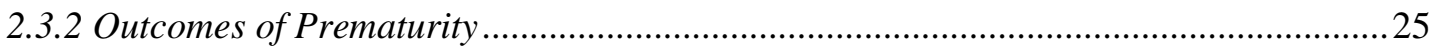

2.3.2 Can bilingualism help premature children's Executive Function Performance? .........26

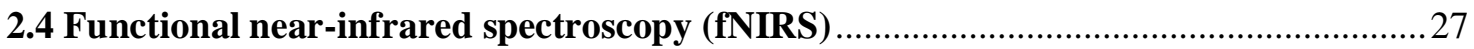

2.4.1 Methodological considerations for studying the infant's brain using fNIRS .................28

2.4.2 Neural origin of cognitive shifting in young children ...............................................29

2.4.3 Bilingualism May Mitigate Set-Shifting Difficulties in ASD Children ..........................29

2.4.4 Probing the early development of visual memory capacity with fNIRS ........................ 30

2.4.5 Prefrontal activation to Stroop interference during development ................................. 31

2.4.6 Evidence for Development of Prefrontal Engagement in Memory............................... 32

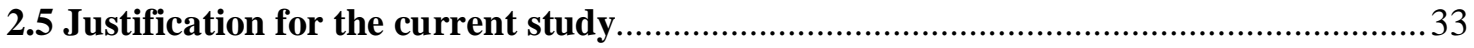

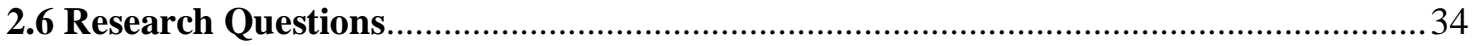

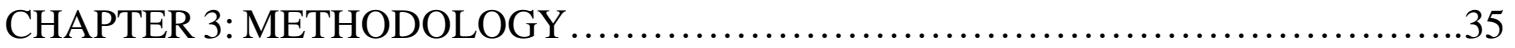

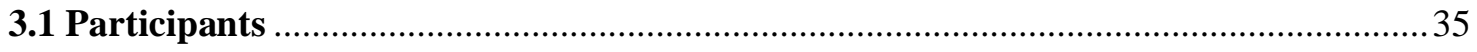

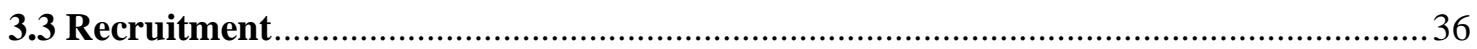

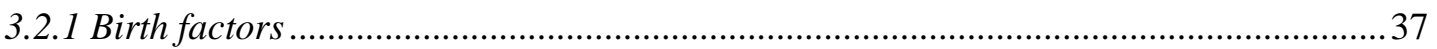




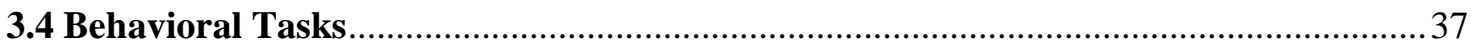

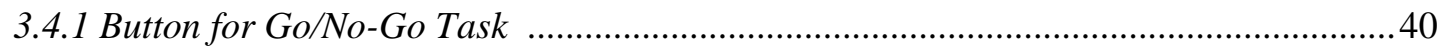

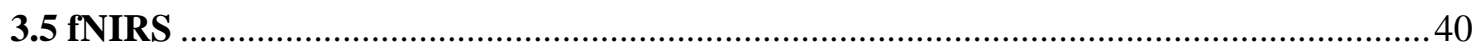

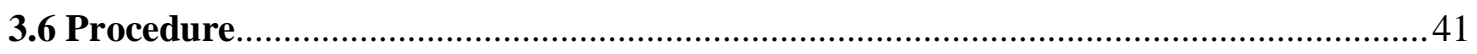

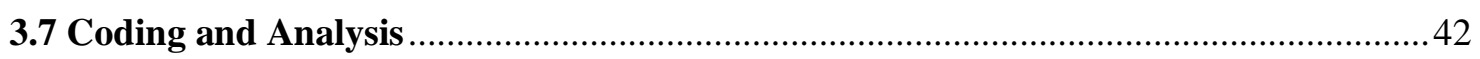

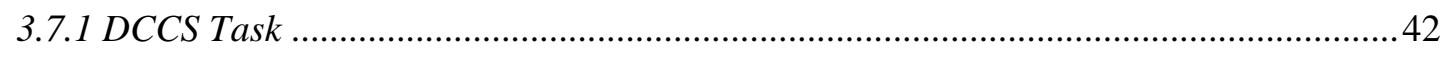

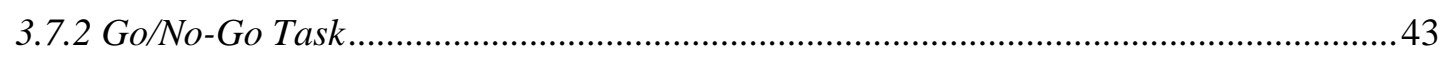

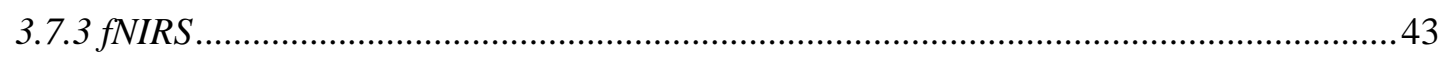

CHAPTER 4: RESULTS ..................................................47

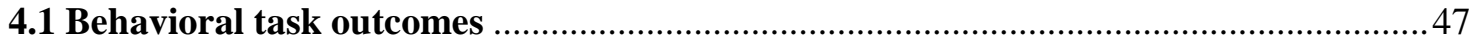

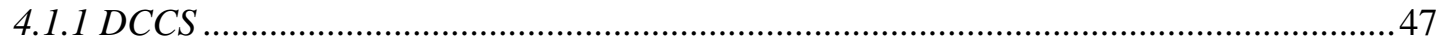

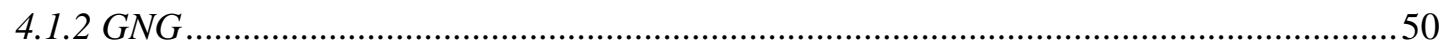

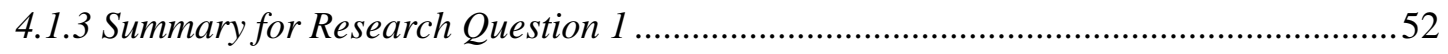

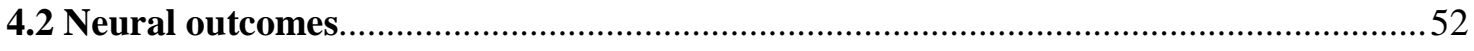

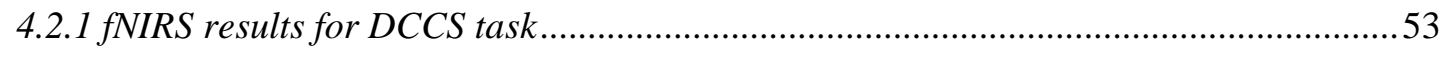

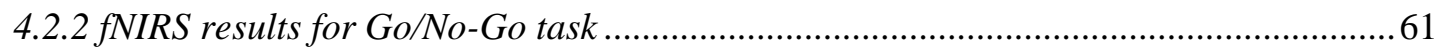

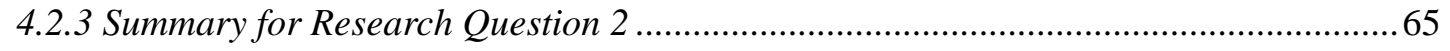

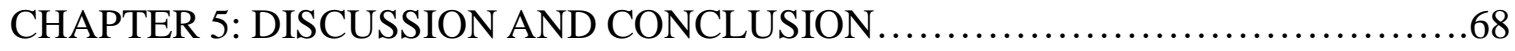

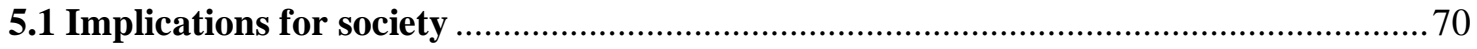

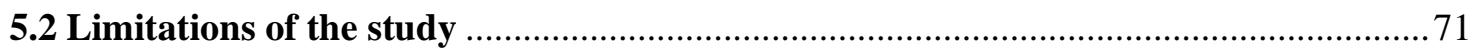

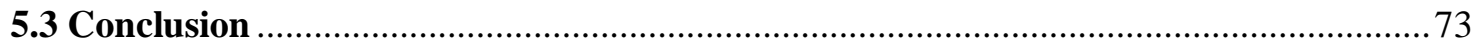

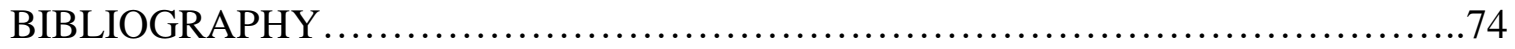




\section{LIST OF FIGURES}

\section{FIGURE}

PAGE

Figure 1: Dimensional Change Card Sort Task Schematic 33

Figure 2: Go No-Go Setup 33

Figure 3: fNIRS Prefrontal Lobe Headband 35

Figure 4: Model of Hemodynamic Response Function (HRF). 38

Figure 5: Electrode Placement and fNIRS Channel connections. 40

Figure 6: DCCS Accuracy for Bilinguals vs. Monolinguals 42

Figure 7 DCCS Reaction Time for Bilinguals vs. Monolinguals: 42

Figure 8: GNG Accuracy for Bilinguals vs. Monolinguals 44

Figure 9: GNG Reaction Time for Bilinguals vs. Monolinguals 44

Figure 10: SPM T-map for oxyhemoglobin of a monolingual participant DCCS. 47

Figure 11: SPM T-map for oxyhemoglobin per language group DCCS ..... 49

Figure 12: SPM T-map for deoxyhemoglobin per language group DCCS 50

Figure 13: SPM T-map for deoxyhemoglobin per language group DCCS 52

Figure 14: SPM T-map for oxyhemoglobin per language group DCCS . 53

Figure 15: SPM T-map for deoxyhemoglobin per language group GNG .55

Figure 16: SPM T-map for oxyhemoglobin per language group GNG. 56

Figure 17: SPM T-map for deoxyhemoglobin per language group GNG 57

Figure 18: Significant beta values with Broadman areas GNG 58 


\section{CHAPTER 1: INTRODUCTION}

Numerous studies have been conducted showing that bilingualism can significantly enhance executive function (EF), an area where pre-term born children struggle (Biran, Verney, \& Ferriero, 2012; Edgin et al., 2008; Head, Baralt, \& Darcy-Mahoney, 2015). Executive function (EF) refers to specific skills, such as attention, emotional control, memory, information processing, cognitive flexibility, inhibition control, and problem solving \& planning. Studies have shown that performance of children born pre-term is significantly lower than that of their term counterparts in school (Edgin, Inder, Anderson, Hood, Clark, and Woodward, 2008; Head, Baralt, \& Darcy-Mahoney, 2015; Moriguchi \& Hiraki, 2013). Children born premature are at a higher risk for disorders, such as autism (ASD), attention deficit disorder (ADD), attention deficit hyperactivity disorder (ADHD), and low EF performance (Park, 2014); however, only low EF performance is the scope of this study.

The early years of life, particularly the time between birth to five years old, is a time of intense and rapid neuronal growth. Infants born premature (especially before 32 weeks gestation) exhibit unexpectedly significant brain dysfunction as they develop, possibly due the significant neurological reorganization that takes place in the second and early third trimester. This is where the fetus is initiating fetal behavioral, individuality, and capacity for extrauterine survival. Many extremely preterm infants will develop psychomotor, cognitive, and attentional function deficits as well as emotional vulnerability and substandard school performance (Als et al., 2004a; Rivkin et al., 2004). Research suggests that these symptoms are due to a central deficit in frontal lobe processing of complex information (Als, Duffy, McAnulty, Rivkin, Vajapeyam, Mulkern, Warfield, 
Huppi, Butler, Conneman, Fischer, Eichenwald, 2004). The ability of the brain to form and reorganize synaptic connections, especially in response to learning or experience or following injury, is known as neuroplasticity. These changes occur in a process called pruning, which is prominent in the first three years, and it is important in neuroplasticity for breaking old connections in the immature nervous system, so that new appropriate connections can be made (Low \& Cheng, 2006). Never again in the life span will the level of brain neuroplasticity mirror that which human beings experience during the first three years of life.

Functional neural correlates for the EF skills groups could be ascertained using a brain imaging technology in different groups of infants born into monolingual or bilingual families. One new imaging modality that is beneficial for research with young children is functional near-infrared spectroscopy (fNIRS). fNIRS has properties that are favorable to functional Magnetic Resonance Imaging, or fMRI, such as its sensitivity to movement in studies that include children. The data collected from the fNIRS are interpreted with the statistical parametric mapping (SPM) analysis in NIRS software, where it localizes the areas of activation based on the preferred montage scheme. There is an extant body of literature that is beginning to use fNIRS to learn more about how children neurally recruit EF (Buss, Fox, Boas, \& Spencer, 2014; Fishburn Hlutkowsky, Bemis, Huppert, Wakschlag, \& Perlman, 2019). However, to date, no study has done so in a premature population, looking at the potential for bilingualism to enhance preterm-born children's EF. With fNIRS alongside behavioral EF tasks, preterm monolingual children and the preterm bilingual children's executive functioning skills can be quantified and compared. 


\subsection{Hypothesis}

The reported linkages between bilingualism and brain differences are convincingly established and well accepted (e.g. Head, Baralt, \& Darcy Mahoney, 2015). For example, it has been identified that there is an increase in density of grey matter in the left parietal cortex of bilinguals relative to monolinguals, and this is more pronounced in those with early bilingual exposure (Bialystok, Craik, \& Luk, 2012). The brain mechanisms associated with the bilingual advantage are functional and plastic, in that they can be developed. And yet, none of this prior work has involved pre-term children, who arguably could benefit the most from the brain changes associated with bilingualism. This study seeks to address this gap by examining whether bilingualism could be a contributor in the enhanced recovery from early brain development deficiencies caused by prematurity.

If this were to be true, then teaching children who are born preterm multiple languages may have a positive influence on their neuroplasticity process and executive functioning skills, bridging the gap for their low EF performance and helping them to do better in school. In 2005, the cost of premature birth to U.S. society was estimated to be just over 26 billion dollars (March of Dimes, 2019). If bilingualism could help children with a history of preterm birth, it is worth exploring. What remains methodologically difficult is how to define bilingualism. Current studies are investigating individuals who do not actively use both languages (i.e. heritage speakers, or people who first learned, e.g., Spanish but then became dominant in English once they started school in the U.S.), to determine if this limits their EF skills, resulting in poorer performance than monolingual individuals (Wang, Zhang, Zheng, Li, Li, Liu, Samudrala, Yu, \& Wong, 2004). One study has even declared that it is not necessary to actively use both languages daily to enhance 
their EF skills (Akhtar \& Menjivar, 2012). Methodologically challenges aside, these studies demonstrate that even minimal exposure to another language could enhance EF. The goal of this study is to understand the differences in neural recruitment of EF between monolingual and bilingual pre-term born children via fNIRS to determine if there are group differences and also, if the differences in localized neural recruitment is one of the many reasons behind their advantage.

\subsection{Rationale}

A significant body of literature has revealed that bilingualism enhances executive functioning skills (Bialystok \& Martin, 2004; Gonzalez-Barrero \& Nadig, 2017; Head et al., 2015). The theoretical rationale is that, because the individual is forced to switch back and forth between languages, they have to ignore irrelevant stimuli in order to pay attention to the language at hand. This daily mental 'exercise' enhances cognitive switching and processing. No study thus far has tested this potential benefit of bilingualism on EF in preterm-born children. Taking advantage of the neuroplasticity experienced during the first three years of life, it is possible that bilingualism may enhance the EF of preterm-born children as well.

\subsection{Significance}

The ultimate goal of this study is two-fold. The first goal is to determine if there are statistical differences in EF task performance between bilingual versus monolingual preterm-born children. The second goal is to determine if bilingual preterm-born children will have statistically different levels of oxyhemoglobin when executive function is recruited than monolingual preterm-born children, as shown by functional near-infrared spectroscopy (fNIRS). Many studies have been conducted to determine EF skills using the 
imaging modality of functional Magnetic Resonance Imaging (fMIRI); however, fMRI is not ideal for studies with young children due to the requirement to stay still for long periods of time. fNIRS allows for neural activity to be recorded while still being able to move and talk.

While technological advancements allow us to keep more preterm-born infants alive, it is imperative that more is done to help these children across the lifespan. Interventions targeting preterm-born children's EF are essential, given that EF is the underlying factor for school success. Many myths abound about bilingualism in U.S. society, due to overall harmful societal messages about the use of languages other than English (e.g. Baralt, Darcy Mahoney, \& Brito, 2017). Neurodevelopmentally, it has been proposed that that the introduction of a second language to preterm children will have harmful effects on their neurodevelopment and will only constrain cognitive performance in school and over the lifespan (Park, 2014). If the current study finds that bilingualism enhances preterm-children's EF, this proposal can be questioned, opening the door for debate in the field about how to improve premature infants' overall cognitive performance. Doing so has implication for clinical outcomes and interventions for these infants. 


\section{CHAPTER 2: LITERATURE REVIEW}

In this chapter, the anatomical areas of the frontal lobe, the prefrontal and orbitofrontal cortices, are reviewed. It is in this area of the brain where executive function is housed. The following sections will cover how and why bilinguals have "enhanced" executive function, the theoretical underlying mechanisms, and EF in children born preterm.

\subsection{Bilingualism}

People with the ability to speak two languages have reportedly performed at a higher academic level compared to their monolingual counterparts (Head et al., 2015). Although bilingualism involves producing in two languages, the bilingual advantage is not only seen in linguistic tasks, but also non-linguistic tasks, such as multi-tasking, memory flexibility, memory generalization, selective attention, inhibitory skills, and cognitive flexibility (Brito \& Barr, 2012; Head et al., 2014; Poulin-Dubois, Blaye, Coutya, \& Bialystok, 2011). These actions lead to better executive function performance, which gives rise to the cognitive advantage, but it is still unknown which executive function skill drives this advantage, if any.

This advantage has been seen in infants as young as seven months old (Kovács \& Mehler, 2009). When introducing multiple languages to a child, they are forced to exercise different executive function skills at a young age, thus accounting for their cognitive advantage (Brito \& Barr, 2012; Kovacs \& Mehler, 2009). It is also suggested that the cognitive advantage bilinguals possess is due to their ability to switch between their two languages, suppressing one language to produce the other (Bialystok \& Shapero, 2005). As an example, a study conducted by (Bialystok, Barac, Blaye, \& Poulin-Dubois, 2010) 
set out to determine the age when the bilingual advantage arises as well as the strategies monolingual and bilinguals use when engaging in word mapping - an executive control skill. Different monolingual groups (e.g. English and French speakers) and bilingual groups (e.g. English + 1 language speakers) were used to make multiple comparisons. The seven tasks used in this study were the Mutually Exclusivity Task, the Luria's Tapping Task, the Opposite Worlds Task, the Flanker task, the Reverse Categorization to assess the children's response inhibition, switching and attention, and inhibitory skills respectively. Children's receptive vocabulary was assessed via the Peabody Picture Vocabulary Test-III (PPVT-III). Participants included 37 French monolinguals, 67 English monolinguals and 56 bilinguals. Results showed that the bilinguals performed significantly better on the Luria's Tapping Task, Opposite Worlds Tasks, and Reverse Categorization Task as compared to both monolingual groups. These findings suggest that the type of language that is spoken is irrelevant - two languages prove to be better than one regarding executive control, which ultimately leads to higher cognitive performance linguistically and nonlinguistically.

\subsubsection{Measuring Bilingualism}

While research consistently shows a bilingual edge, one methodological problem is how researchers can properly measure or categorize a bilingual person. For example, if a participant is categorized as a bilingual, but only has receptive knowledge of the language

and cannot produce in the language, that makes a great difference (Montrul, 2013; Torres, 2013). Persons with receptive knowledge only of a second language should not be grouped with fully productive bilinguals. Their performance can affect the bilingual group's average scores, thus not accurately representing the true data, or an exposed individual is 
listed as a monolingual, in which case, both can result in a Type 1 Error. There are many different influences that affect an individual's language skills and cognitive functioning, whether it is environmental or genetics, so importance should be stressed on considering outside factors that may inaccurately alter results. For this reason, a variety of means should be employed to determine bilingual status. This can be done via questionnaires administered to participants and to parents to get an idea of children's exposure to the language(s), parents' level of education and occupational status, as these factors seem to influence the bilingual advantage. Standardized vocabulary tests can also give insight into participants' bilingual capacity in the second language. However, one easy way to confirm productive capacity is to ask in the language background questionnaire what language the child purported to be bilingual responds in when addressed in Spanish (the second language). If the child and the parent confirm that the child always, consistently, and comfortably responds in Spanish, this may methodologically be one means to confirm their productive capacity. The next sections will highlight the skills that give rise to the advantage that separates a bilingual from a monolingual.

\subsection{Executive function}

Daily, people must plan out their tasks, make decisions, solve complex problems, and remember steps to complete key tasks. These daily processes are carried out in the frontal lobe and are referred to as one's EF skills. EF includes inhibition, attention, cognitive flexibility, democratic disposition, information processing, memory, and metalinguistic awareness (Bialystok, Barac, Blaye, and Poulin-Dubois, 2010; Buchweitz \& Prat, 2013). Each skill sets out to execute specific tasks, but deficiencies in these areas can make it difficult or impossible for an individual to execute the tasks in comparison to 
an individual without deficiencies. Thus, it is evident that EF is important for school because these skills are the dominant skills necessary for academia. Poor EF will inadvertently result in poor school performance. EF can be measured by tasks that were created to test the performance of specific executive functioning skills. Bilingual individuals have been shown to have enhanced executive function (Bialystok et al., 2010; King \& Mackey, 2007; Poulin-Dubois et al., 2011; Prior \& Macwhinney, 2010). However, the question remains: what mechanisms enhance their executive function?

\subsubsection{Problem Solving \& Planning}

Problem solving, and planning is the ability to identify and coordinate information to complete a task and is another key aspect of EF and key to success in school. Individuals with difficulties in this area may have trouble completing assignments due to unsuccessfully identifying or gathering the necessary information according to the task at hand. For example, in math word problems, the first steps are to identify the clue word that would suggest addition, subtraction, multiplication, or division. Deficits in this area may lead to the child having difficulty identifying the clue word, which would imply that a specific math function be performed.

In a study that examined how children problem solve and use strategies to overcome various tasks, McCarty, Clifton, \& Collard, (1999) observed 36 children - 12 at each age group 9, 14, and 19 months - complete a variety of age appropriate tasks that required problem solving. The researchers studied how the children overcome the obstacles presented to them. The first task consisted of the children feeding themselves with a spoon after their parent fed them to make them feel comfortable eating. The investigator oriented the spoon to the right for one trial and left for the next, to see how the child would respond 
to the awkward positioning of the tool to eat the food. The second task consisted of a similar procedure, but instead involved a toy with a handle. Results showed that the younger children reached for the objects with their dominant hand regardless of its orientation, whereas the older children made the corrections to properly use the tool. The findings from this study showed that with age ( 5 months) the children were able to complete the given tasks like an adult, and they solve problems through trial and error and past experiences. In sum, the studies on problem solving, have a common characteristic of paying close attention to the task at hand in or to complete it efficiently (Cunha, Babik, Ross, Logan, Galloway, Clary, and Lobo., 2018).

\subsubsection{Attention Control}

Attention control, another aspect of EF, is the ability to stay focused on a task despite possible interfering stimuli. It can be categorized into bottom-up attention which deals with attentional guidance by external stimuli, and top-down attention which deals with internal guidance based on experiences, knowledge, or goals (Katsuki \& Constantinidis, 2014). An example of someone with attention control problems are those diagnosed with Attention Deficit Disorder (ADD) or Attention Deficits Hyperactive Disorder (ADHD). This makes it difficult for the individual to concentrate without losing focus on the task at hand. Typically, children are diagnosed with these disorders when an authority figure notices certain characteristic, like not completing a thought before moving on to the next.

In a recent two-study experiment, Comishen, Bialystok, and Adler (2019) examined differences in attention control between full term, healthy 6-month old monolinguals and bilinguals using an eye tracking device - Visual Expectation Cueing 
Paradigm (VExCP) - to track the children's eye movements for comparison. In study 1, two events were presented, a randomized block and a predictable block. The infants were given a cue at the center of a monitor for a few moments before it disappeared and reappeared in either the left or right lower corners. The infants' task was to correctly anticipate the location, in which the cue would reappear for both blocks. Results showed that the monolinguals and bilinguals both performed the same in for the random and predictable blocks. In study 2, variations were made for the Visual Expectation Cueing Paradigm where there were only two predictable events. The monolinguals made correct anticipations at a greater rate than the bilinguals in the pre-switch task, while the bilinguals did better in the post-switch task and made more frequent correct anticipations than by chance for both pre- and post-switch tasks. The reactive latencies were recorded and showed that the monolinguals took a bit longer than bilinguals in the pre-switch phase and were statistically significant, being slower in the post-switch phase. In other words, the bilinguals were faster. These findings prove that attention and selection play an important role in the cognitive benefits of bilingualism. Selection plays into the cognitive skillset because it relies on inhibition, which is thought to be one key underlying mechanism behind the bilingual advantage.

\subsubsection{Inhibition Control}

Inhibition control, a key aspect of EF, plays a common role in many studies of bilingualism as one of the underlying mechanisms behind enhanced cognitive performance. The reviews below expands on inhibition control in bilinguals versus monolinguals, and if the cause of the bilingual advantage is due to their ability to voluntarily suppress or evoke certain physiological processes, or if it is just one of many 
mechanisms. Teaching children multiple languages in a balanced, consistent manner seems to be another underlying mechanism that improves executive function skills, leading the way for better cognitive performance (Brito \& Barr, 2012; Comishen, Bialystok, \& Adler, 2019). However, these factors alone cannot explain the processes that occur to give bilinguals this advantage over monolinguals, which has mainly been seen in academia, further leading to economic success.

Bialystok and Shapero (2005) reported on two studies set out to differentiate between monolingual versus bilingual executive processes that each group experiences using the various tasks: digit span, children's embedded figures, ambiguous figures, opposite worlds, and Raven's colored progressive matrices, which tests working memory, selective attention, inhibitory control, cognitive switching, and nonverbal intellect respectively. Inhibition control grouped into response inhibition and interference suppression is a skill better executed by bilinguals than monolinguals as young as 5 years of age. In their study 1, a total of 48 six-year-old children, half bilingual half monolingual, participated. Results revealed that the bilinguals did better but not statistically significant compared to the monolinguals in the figure-ground and content-meaning reversal of ambiguous figures tasks. However, the processes which they used to unmask the figures were different. In their study 2 , bilinguals outperformed the monolinguals on all tasks; however, the results did not show statistically significant differences between the two groups for the DCCS pre- or post- trials. Due to the bilinguals outperforming the monolinguals in tasks that did not rely on inhibition control, it cannot be considered the sole factor underlying the bilingual's ability to unmask the ambiguous figures. 
In a related review, Akhtar and Menjivar (2012) explored the correlations between cognition and language in children who were exposed to multiple languages at an early age, by reviewing multiple studies within this field of study. The authors point out that the biggest misconception that is being advertised by physicians to parents is introducing a second language at an early age causes cognitive delays, or cognitive deficiencies. Most research in this area of study can attest for the bilingual advantage that is seen when compared to monolingual peers; however, there is also evidence that more than just vocabulary and inhibition control need to be considered. This article takes into consideration certain external factors such as socioeconomical status (SES), the amount of exposure to each language and the environment in which they are exposed, as well as interpreting the difference between delays and deficits. In sum, Akhtar and Menjivar (2012) express how trivial the delay in speech young bilingual children face is compared to the outcome of a completely bilingual adult. According to Saiz and Zoido (2005), the average college-educated bilingual adult is more successful than a college-educated monolingual even though the monolingual did not experience any delays in early childhood. 


\subsubsection{Cognitive flexibility}

Cognitive flexibility is the ability to change from one mindset to another, and also underscores EF. If a child has cognitive shift problems, they may not be able to switch between multiple instructions efficiently. This switching ability can be tested using the functional behavioral tasks that are designed to target cognitive flexibility by forcing the individual to switch through multiple rules to complete one task. The following review will discuss cognitive flexibility and highlight some inconsistencies in literature based on whether bilinguals rely more on inhibition or cognitive switching.

Prior and Macwhinney (2010) set out to challenge the concept that inhibition is the major factor in the advantage bilinguals possess. Their interest was on the promotion of cognitive flexibility as an underappreciated factor in enhancing cognitive performance. They present a new perspective on the uncertainty of inhibition being the underlying factor of the bilingual advantage. Many studies suggest suppression of the language as the cause and neglect to consider that it may be the switching of the mental set entirely to the next language. Prior and Macwhinney (2012) determine mixed costs and switching costs of bilinguals and monolinguals using tasks that closer relate to mental switching instead of inhibition. To determine their hypothesis, they recruited 47 bilingual and 45 monolinguals 18 \& 19-year-olds to partake in the various tasks: shape/color decision, Peabody Picture Vocabulary Test - IIIL, Dunn and Dunn, Language History Questionnaire, Task switching Paradigm, and Operation Span Task. A two-way ANOVA revealed results that coincide with literature: monolinguals outperformed the bilinguals in the Picture Peabody English Vocabulary Test. They also had faster reaction times, except for the mixed switch task. However, despite faster times, the bilinguals performed significantly more accurately on all aspects of the single-task blocks and mixed task blocks. Based on these findings, it can 
be said that cognitive flexibility relies on aspects of inhibition, but also rely on attentional control, as proposed in the previous section. The next section will discuss how information is processed between full-term infants and pre-term infants.

\subsubsection{Information Processing (IP)}

Information processing, determined by accuracy and speed, is the ability to perform a learned task. If a child has information processing deficits, they may not be able to complete a given task that was previously learned. For example, an instructor is explaining algebra to his students and asks if they understand what has been covered thus far, and the students answer "yes". Unconvinced, the instructor selects one of the students that answered to try an example on the board. As the student tries to replicate the similar problem, a pause occurs. It appears the example the instructor did on the board did not fully process with the student. However, repetition is a major factor in information processing. It cannot be expected after being introduced to new material without repetition to completely grasp the concepts to perform that task. If multiple repetitions outside of the initial reps of the process being taught is needed, it can be suspected that information processing is poor compared to an individual that may fully understand with fewer reps. The following study will further elaborate how information processing (IP) compares between preterm children and full-term children.

\subsubsection{Memory}

Memory is categorized into short-term memory and working memory. Short-term memory is the ability to contain information for a small period, while working memory coordinates the information in the short-term memory, which is necessary for solving problems. Since executive function (EF) skills are controlled by the prefrontal lobe, it 
contains connections to the hippocampus. The hippocampus is a region of the brain responsible for short and long-term memory and consolidating memories creating relational representations. For a bilingual, relational representations are stronger in comparison to monolinguals since they must connect two words from different languages and link them to one referent. (Brito \& Barr, 2012). According to Britto and Barr (2012), bilingual infants as young as 12 months show signs of memory generalization for colors, and infants as young as 18 months have shown generalization for shapes. The following study compares the memory generalization of bilinguals to monolinguals.

Brito and Barr (2012) exposed children to different action targets to prove if bilingualism rears specific cognitive advantages over monolingual children. A total of 42 infants were included in this study and divided into the generalization condition or baseline condition. There were 15 monolinguals, 15 bilinguals, and for the baseline six monolinguals and bilinguals. In the generalization condition, the infants were given three targets: pulling off a mitten, ringing the bell on the mitten, and replacing the mitten as a demonstration before testing. For the baseline condition, the infants were only exposed to the three targets during the trial with no previous presentation. The results revealed that nine of 15 of the bilingual children compared to one monolingual, successfully imitated the target actions, exhibiting their higher capacity of memory generalization, which coincides with the findings of similar studies. Some possible explanations for the higher performance of the bilinguals are selective attention and more developed mental representations than monolinguals. This capacity is theorized to come from their experience of having been exposed to two languages. 


\subsubsection{Metalinguistic Awareness}

Another area in which brain benefits of bilingualism are seen is in the area of metalinguistic awareness. Metalinguistic awareness refers to the ability to think and talk about language as a subject itself, and can comprise phonological, lexical, and syntactic awareness. Phonological awareness focus on sound structure which helps coordinate with reading; lexical awareness is based on the concept that words are arbitrary and can have different terms for one referent, as previously mentioned; and syntactic awareness involves knowing the rules associated for completing grammatical sentences (Akhtar \& Menjivar, 2012). The following study summarizes various studies conducted with monolinguals and bilinguals to investigate EF, Theory of Mind (TOM), vocabulary size, word learning, lexical access, pragmatics, and metalinguistic awareness, which will be the focus of this section.

Akhtar \& Menjivar (2012), compared multiple studies conducted with school aged monolingual and bilingual children to assess the differences in awareness by using the Piaget's sun/moon task, phonological awareness task, two-word awareness task, and symbol substitution task. An advantage was seen for bilinguals in the phonological awareness task, but this advantage seemed to regress with age as learning to read for the monolinguals compensated for their lack of the advantage. The bilingual advantage was also seen in the word awareness assessment as inhibition may have been the underlying mechanism for this task. The bilingual children also performed better on the test measuring syntactic awareness as well. Based on the various studies combined, the researchers put forth three key take-aways. First, they argued that proficiency in both languages is necessary to fully reap the benefits of bilingualism - except for word awareness, exposure 
to two languages is better than one - no matter the level of exposure, and higher proficient bilinguals outperform lesser proficient bilinguals. There is controversy over the level of exposure to a language one needs to reap bilingual benefits, but more research must be conducted.

To summarize, bilingualism is reliably associated with attentional control and executive function, working memory, metalinguistic awareness, and abstract and symbolic representation (see meta-analysis by Adesco et al, 2010). Bilingual children have an enhanced capacity to control their use of knowledge, and this is especially the case when distracting stimuli must be ignored. These enhanced executive processes are developed earlier in bilingual children than in monolingual children. The daily experience of controlling attention to one language, while suppressing information from the other active language that is not relevant to the task-at hand, appears to boost bilingual children's cognitive function. These advantages are associated with physical changes in the brain that stay with bilinguals for a lifetime. Importantly, the brain mechanisms associated with the positive benefits of bilingualism are plastic, and can be developed even with early bilingual immersion schooling

\subsubsection{Understanding Across Cultures}

Many researchers have argued that bilingualism also helps children to see others more fairly and equitably. Bilingualism opens the door for many cognitive skills, but one skill that is overlooked is the ability to be more open and aware of other cultures and how they operate. King and Mackey (2007) elaborate on the effects of knowing two or more languages on an individual's cultural awareness simply because how they form grammatical sentences. They report that children who speak multiple languages innately 
understand that life for people of different languages bring different cultures and views of life and that is they are neither superior or inferior, just different.

\subsection{Preterm-Born Children}

A healthy term pregnancy falls between 37 to 40 weeks. According to the Center for Disease Control and Prevention (CDC) and the World Health Organization, children born earlier than 37 weeks are considered moderate to late pre-term; children born before 32 weeks are considered very pre-term and usually weigh less than 1,500 grams at birth; and children born earlier than 28 weeks are considered extremely pre-term and usually weigh less than 1000 grams (Eichenwald \& Stark, 2008). In 2017, March of Dimes reported 9.8\% premature births for the United States and 10.6\% for Miami, Florida alone (Dimes, 2017).

Many of these children will experience complications such as developmental delays, growth reduction, cerebral palsy, lesions in the brain, vision and hearing impairments, and cognitive deficiencies in areas such as executive function (Biran et al., 2012; Edgin et al., 2008; Hackman, Gallop, Evans, \& Farah, 2015; Kolb \& Gibb, 2011; Rysavy \& Ehret, 2019). Pending the source, $25-50 \%$ of pre-term and low birth weight infants exhibit developmental disabilities related to cognition, behavior and motor activity, with school disturbance being a nearly uniform result (Volpe, 1999). By school age, poor academic achievement may become evident in children without obvious neurodevelopmental impairments. It has also been well documented that low birth weight and pre-term infants have a high incidence of perinatal brain injury and have high rates of poor school performance. The pathogenesis of brain injury in pre-term infants is complex 
and as a field, we do not yet have a thorough understanding of it nor of its consequences. The principal brain lesions that underlie much of the neurologic manifestations seen in preterm infants are intraventricular hemorrhage (IVH) (and its accompanied complication, periventricular hemorrhagic infarction) and periventricular leukomalacia (PVL) (Volpe, 1999). The developmental sequalae of these lesions include poorer cognitive function and academic performance when compared to normal birth weight controls.

Another type of lesion seen in preterm born infants is cerebellar hemorrhages $(\mathrm{CBH})$. One study reports that $\mathrm{CBH}$ occurrence is inversely related to birthweight (Limperopoulos et al., 2005). However, the underlying mechanism behind these lesions is not yet completely understood (Biran et al., 2012; Limperopoulos et al., 2005). A characteristic seen in premature infants is reduced cerebellar hemispheric volumes compared to term infants, where statistically significant differences in structure can be seen in their white matter, corpus callosum, and ventricles (Edgin et al., 2008). These differences are derived from neuroimaging devices (e.g. MRI, NIRS, PET, CT), which can be used to determine how the injury affects cerebellar volumes (Biran et al., 2012). Of concern is the effect of these injuries on preterm children's executive function. Neuropsychological testing reveals that they perform more poorly on measures of attention, executive function, memory, spatial skills, and motor function (Hack, 2000). The cognitive processes associated with executive function include deployment of attention, working memory, utilization of feedback, planning and organization, goal selection, and self-regulation. The interrelated processes that make up executive function are responsible for a child's cognitive functioning, behavior, social interaction, and emotional control. The relevance of these deficiencies lies in the way in which they affect a young child's school 
readiness. As stated earlier in this thesis, EF is the main underlying factor of a child's school performance and success. Given preterm-born chldren's risk for significant problems in the area of EF, they are also set up to have difficulty in school

\subsubsection{Effects of Prematurity on Executive Function}

The last two months of gestation are crucial for a child's development since it is the period when the prefrontal lobe matures - the area of the brain that controls EF. The high risks developmental disabilities that occur in premature infants can be linked to poor school performance. Many of these disabilities can manifest in higher order functioning delays, including challenges with executive function. There are several effects associated with prematurity in executive function, which include inhibition, attention, memory, cognitive flexibility, information processing, problem solving and planning. A study conducted with children who had specific language impairment (SPI) showed a positive correlation between inhibitory control and working memory be determined (Marton, Kelmenson, \& Pinkhasova, 2007).

Rose, Feldman, and Jankowski (2009) investigated information processing in toddlers to determine if they experience continuity or discontinuity, and if the deficits in premature born children persist, or if they "catch up" to a full-term individual's developmental level. They worked with 144 full-term and 59 pre-term infants in the follow up stage (24 and 36 months) as an extension of another study. Memory, processing speed, attention, and representational competence were assessed using various familiarization tasks, visual expectation paradigm (VExP), and Visual Paired - Comparison (VPC). Results revealed that the preterm children's short-term memory, recall, accuracy, attention, and processing speed were statistically significant, being significantly poorer at both $24 \&$ 
36 months compared to the full-term children. Four findings came about from this study: preterm born children's deficits last throughout the lifespan and do not "catch up" to the developmental levels of a full-term born child, statistically significant continuity was seen from infancy to toddlerhood, cognitive skills are additive to account for global cognitive abilities, and IP proves continuous over the first 3 years of life. These deficits have been investigated for information and solutions on the effects a preterm birth has on executive function.

In a study by Orchinik (2014), the extent of the effects a premature birth has on executive function was investigated in a kindergarten sample of 147 premature born children and 111 term-born controls, and the degree of premature birth or neonatal complications are predictors of EF. This study highlights the importance of valid EF assessments, the differences in extremely premature (EP) children and the different factors that play a role on their EF and non-executive functions. EF performance is associated with intelligence (IQ), but Orchinik expresses the importance of determining what aspects of EF contribute to overall intelligence. In theory, intelligence can be broken into broad intelligence and narrow intelligence. Narrow intelligence is specific, while broad includes two factors, Gc (measure of acquired knowledge) and Gf (fluid reasoning ability). In this study, Gc was used as the control for cognitive ability and is used to help minimize general intelligence functions. The EF tasks that were chosen for this study include, Nebraska Barnyard task for working memory, Go/No-Go for inhibition control, Shape school for different measures of inhibition, and Trails-Preschool for cognitive switching, inhibition control and processing speed. Results showed that 1) children that were born premature had EF deficits which also was affected by neonatal risk factors 2) deficits were noticed in 
working memory, task switching, and inhibition 3) the Nebraska Barnyard task revealed that working memory is vulnerable in EP children with bronchopulmonary dysplasia 4) despite results of similar studies, results showed that birth weight was not directly associated with EF deficits 5) lastly, higher socioeconomic status resulted in higher EF performance (Orchinik, 2014).

A review by Taylor and Clark (2016) also addresses aspects of EF which include self-regulation, self-control, and emotional control, which are related to academic performance, health, and wealth (i.e. socioeconomic status). Development and risk factors of $\mathrm{EF}$ are discussed along with ways to mitigate the $\mathrm{EF}$ assessments that are given and ways of intervention. The main components of EF - working memory, inhibition, and cognitive flexibility - can be affected by many external factors including neonatal complications, neural abnormalities, and the environment. For instance, attentional control skills are strengthened when external stimuli demand attention, which is an aspect of cognitive flexibility. However, there are also internal factors that play a role on EF. Across literature, long range white matter connectivity has been associated with higher EF performance, which is assumed to be a result of pruning and myelination (Taylor \& Clark, 2016). White matter volume for preterm children is considerably less than that of their term born counterparts; though, impairments of EF vary with the degree of prematurity. There are three consistencies seen across literature: as preterm children age their verbal fluency skills decrease, while their selective attention and response inhibition skills increase, different neural network processes exist between preterm and term born children, and the gap in EF between normal birth weight and low birth weight children increases over time. It is also important to note that not all neural impairments are associated with poor EF due 
to "neural compensation" - neuroplasticity - which makes up for the neural impairments, which is why EF is not affected in all preterm children (Taylor \& Clark, 2016).

It is important to highlight that the integrity of white matter tracts in the brain is a determining factor of EF performance. A study by Woodward, Clark, Pritchard, Anderson, and Inder (2011), also explores white matter abnormalities to determine its relation to EF deficits by comparing very preterm children and term born children. Diffuse non-cystic white matter abnormalities (WMA) are the most present form of brain insults that affect preterm children, along with enlarged ventricles, white matter volume loss, delayed myelination, and thinning of the corpus callosum. The WMA are detected via diffusion tensor imaging in supplement to MRI to validate findings. Participants included 110 very preterm and 113 term born four-years-old, all born at Christ Church Women's Hospital. The children were given four different EF assessments which were Tower of Hanoi for planning, Flexible Item Selection Task for cognitive flexibility, Visual Search for selective attention and organizational skills, and Shape School for inhibition and cognitive flexibility. The statistical analyses used were: an ANOVA and a MANCOVA - to compare performance, Mantel Haenzel Chi squared statistic - for linear trends, and multiple regression model - to predict the global outcome for EF. Results showed that $58 \%$ of children born very preterm had moderate WMA suggesting the lower IQ compared to term born children. The results of the Tower of Hanoi, Visual Search, and Flexible Item Selection Task (FIST) showed a statistically significant difference in performance between the groups. However, the Fist was not statistically significant when controlling for SES and the Shape School task did not return statistically significant differences between the groups. The results of the study presented that EF composite score, WMA and gender are all 
moderate predictors of EF outcome.

\subsubsection{Outcomes of Prematurity}

Another outcome of prematurity is life-long deficits in EF. In a study by Kroll, Karolis, Brittain, Tseng, Froudist-Walsh, Murray, and Nosarti (2017), it was determined if the EF deficits a preterm child experiences persists over the life-span, and if these deficits have a negative influence in real life aspects, such as a career, which affects overall wealth, social behavioral skills, and health, which can reflect poor EF. The study included 122 very preterm adults between 28-34 years of age, who were born at the University College Hospital London and 79 term-born adult controls that weighed > 2500 grams. Individuals with any neurological conditions, brain injury, or other insults that may sway the results of the study were excluded. Methods included collecting sociodemographic information for all participants, as well as IQ scores via the Wechsler Abbreviated Scale of Intelligence. To determine real-life achievement participants were given the Role Functioning Scale (RFS) assessment to measure social and working skills. A t-test was used to measure sociodemographic and cognition in addition to SPSS 22.0 and Matlab 13b, an ANCOVA for comparison of the two groups, while controlling for sex and age, and multiple regressions to determine the connection to real-life achievements and EF. Results showed that very preterm adults were statistically significant and inferior to the performance of term-born adults. Very preterm adults spent less time in school and as a result have poorer

performance in education, lower rates of employment, and lower GRFI scores compared to the term group. In short, results were as expected, very preterm-born adults showed positive correlation between real-life achievements and EF, while term-born adults did not. Ultimately suggesting that EF deficits persist throughout the lifespan because of time spent 
in educational facilities, neonatal perinatal injuries, and sociodemographic status (Kroll et al., 2017).

\subsubsection{Can bilingualism help premature children's Executive Function Performance?}

From the extensive research that has been conducted and the literature reviewed above, there is no doubt that bilingualism enhances executive function in children with and without cognitive deficits such as autism (Gonzalez-Barrero \& Nadig, 2017; Orchinik, 2014; Park, 2014). It is therefore reasonable to assume that these benefits will be extended beyond healthy, "normal" individuals. Therefore, children born preterm should also benefit from learning a second language, strengthening their neural connections in the frontal lobe, hence strengthening EF. Surprisingly, there is only one review to date that proposes this as a possible solution. Head et al. (2015) looks to bilingualism as a mitigation to the cognitive deficiencies that are present in very premature born children. Their paper was a review and a theory paper, however, and not an empirical one testing out their proposed hypothesis. More research must be conducted to support the idea that bilingualism may help prematureborn children in the area of EF. The current study experimentally tests the idea proposed by Head et al. (2015) by empirically comparing the EF task performance of monolingual versus bilingual preterm-born children (taking great care to only include fully productive bilinguals given the implications in the literature for operationalizing bilingual status). The present study also incorporates a new technology, functional near-infrared spectroscopy (fNIRS), to determine the time differences in hemodynamic responses between the preterm bilingual and monolingual group, as this should expose the truth behind these claims. 


\subsection{Functional near-infrared spectroscopy (fNIRS)}

Functional near-infrared spectroscopy (fNIRS) is a non-invasive imaging modality that assesses brain activities through the changes in cerebral hemoglobin oxygenation and concentration (Farras, 2009). fNIRS determines these changes by converting the optical density (OD) to oxy/deoxy-hemoglobin (hbO/hbR) by applying the Modified Beer Lambert Law (MBLL) (Kocsis, Herman, \& Eke, 2006). Typically, an fNIRS system utilizes fiber optic cables to transmit light between the light sources/detectors and a headband. The light emitted from a NIR light source (i.e. $650-950 \mathrm{~nm}$ ) is conducted to a specific region of the head using the illumination fiber, and the resulting diffuse reflection is collected using emission collection fibers and then transmitted to the photodetectors. The responses of the photodetectors are used to determine the levels of oxygenated and deoxygenated hemoglobin in the brain specific to the region of interest (ROI) are calculated. The depth of the investigation of a NIRS system is determined by the distance between the illumination site and the detection site (Kopton and Kenning, 2014). For fNIRS, the optimal distance is two to three centimeters (Kopton \& Kenning, 2014).

The use of fNIRS has increased due to its non-invasive feature, better temporal resolution, lower cost, portability, and independent use. fNIRS does have some disadvantages. As compared fMRI (functional magnetic resonance imaging), fNIRS yields a weaker signal to noise ratio (SNR), can create false positives, has a lower spatial resolution, and cannot measure the deeper areas of the brain (Cui, Xu et al. 2011; Moriguchi and Hiraki 2009). However, a major positive aspect of the fNIRS technology is that it is less susceptible to movement, records real-time neural signals, and is completely noninvasive. It is also significantly less expensive; fMRI procedures mandatorily require an 
expert technician on site. The false positives that can be associated with fNIRS technology can be mitigated by using a technique known as principal component analysis (PCA) (Cui, Xu et al. 2011). So far, only a handful of studies have used fNIRS to examine the neural recruitment of EF in children. These studies will be reviewed below.

\subsubsection{Methodological considerations for studying the infant's brain using fNIRS}

Aslin (2012) provides a detailed synthesis of the use of fNIRS and different measurements of neural activity in the infant's brain. The researcher also highlights important methodological techniques as well as potential limitations that researchers need to consider when using fNIRS on young children. So far, most of this area of research has explored the way that infants respond to auditory stimuli. For example, Pena, Maki, Kovacic, Dehaene-Lambertz, Koizumi, Bouquet, and Mehler (2003) found that there was more activation in the left hemisphere when infants were presented with recordings of forwarding speech rather than backward speech (Aslin, 2012). Another line of research as summarized by Aslin (2012) is visual stimuli and the way in which the brain responds to it as measured by NIRS. He highlights some literature reviews of studies conducted using NIRS and techniques that can increase the NIRS' results reliability. The central questions presented were, "Do the channels provide brain-specific information and, how are NIRS activations distributed over cortical areas known to be involved in particular sensory modalities?" Two designs were presented: hypothesis-driven prediction from adult data and exploratory research. The researchers concluded by explaining that multiple factors contribute to the reliability of NIRS data for infants such as the placement of the probes, re-testing for different probe placement, and estimating surface noise to improve the signal to noise ratio. 


\subsubsection{Neural origin of cognitive shifting in young children}

Moriguchi and Hiraki (2009) used fNIRS to determine what parts of the brain attribute to cognitive shifting in adults and children by also incorporating the Dimensional Change Card Sort task. A total of 36 participants underwent the study. These included three groups consisting of ten adults, 12 five-year old children and 15 three-year-old. The DCCS task was administered to all participants. This task requires the participant to accurately sort the various cards (e.g., red star, blue cup, red cup, blue star) according to their color or shape depending on the specific rules that are given. The responses of everyone were recorded via video recorder; accuracy was scored via scoresheet. The reaction time of everyone was recorded by obtaining the average time it took to sort one card. The behavioral results of this study suggest that children at the age of three years had difficulty performing in the pre-switch and post-switch phases. However, children at the age of five years had no problem performing in the pre-switch and post-switch phases. Surprisingly, the five-year old's' performance resembled that of the adults. The NIRS results showed that adults had statistically significant activations in the bilateral inferior prefrontal cortex

during the post- and pre-switch phases. When comparing the recordings to the control phase, it was noticed that the right and left inferior prefrontal areas had statistically significant increases in oxygenated hemoglobin.

\subsubsection{Bilingualism May Mitigate Set-Shifting Difficulties in ASD Children}

Barrero and Nadig (2017) experimented measuring multiple variables such as reaction time, set-shifting, and language skills to determine if bilingualism benefits children with autism spectrum disorders (ASD). A total of 40 participants were included in the study: 20 typically-developing children and 20 children with Autism spectrum 
disorder. The children performed the DCCS task (computerized and non-computerized), which is designed to determine the child's cognitive flexibility and information processing skills. Bilingual children with ASD performed better than monolingual children with ASD and no differences occurred regarding working memory among the ASD children. However, it was noted that the bilingual children outperformed the typically-developing children in the DCCS task on the more complex border dimension. Some possible explanations for these findings are given. One of these is that children with ASD perform better than typically developing children in the computerized version of the DCCS task because it removes a social aspect, whereas TYP children played better with an experimenter because of the interactive social element. Thus, the relevance of this study is to inform families with children who have ASD, to introduce bilingualism as a beneficial factor in their neurodevelopment.

\subsubsection{Probing the early development of visual memory capacity with fNIRS}

In a different study by Buss, Fox, Boas, and Spencer (2014) used fNIRS to gain an understanding of visual working memory (VMW) in the early stages of life. The goal was to investigate the limitations of the working memory capacity in young children and identify any regions of the brain that may underlie the visual working memory deficits in EF. Participants were 28 3.5-years and 19 4.5-years (Asian, African-American, and Caucasian children). Each participant was instructed to play the matching card game. Flashcards with different shapes shown to the children for about two seconds. The participants were asked to remember the card shown, and after a short delay, a new flashcard was presented to them. The participants' job was to identify if there were any changes to the card. Three practice trials were conducted with the flashcards, and four 
practice trials were on the computer to ensure the child completely understood the procedure. A total of four turns were completed by each child, where each run consisted of six same and six changing trials totaling 12 trials. The behavioral results show that the 3.5 age group had a VMW capacity of about 1.5-2 items, 5-year old had a VMW capacity of 2-3 items, and 7-year old's had a VMW capacity of about four items. The fNIRS results showed that the frontal and posterior cortical regions contributed to the VWM; however, it is still unsure if these were the only areas that underlie the VMW. This study sheds light on the areas related to VWM.

\subsubsection{Prefrontal activation to Stroop interference during development}

Schroeter, Zysset, Wahl, and Von Cramon (2004), also explored EF and its neural origins with fNIRS and children. The researchers used the Stroop interference task to stimulate brain activations in executive function in the frontal lobe. The Stroop interference task consists of a participant correctly identifying the word or color presented on a screen. For example, the word RED may appear in green ink, and depending on the specific task, they must identify the color and not the actual word and vice versa. This task requires inhibition skills to respond to the appropriate task. As the brain develops, Stroop interference reduces implying that the brain is maturing and increasing in inhibition skills. A total of 23 children and 14 young adults were included in this study. The requirements included native German speakers, normal vision, right-handed, no mental disorders, no prescribed psychotropic medication, and no intelligence deficits. Thirty total trials consisting of ten neutral, ten incongruent, and ten congruent conditions were given to each participant with only four seconds to respond with 12 -second intervals. At a sampling frequency of six Hz, the oxy- and deoxy- and total hemoglobin changes were measured. 
Behavioral results showed that the Stroop interference for reaction time was higher in children than adults, supporting Daniel et al. (2000) study.

fNIRS results showed that in the left lateral prefrontal cortex of the children, oxyhemoglobin increased, and deoxyhemoglobin decreased during the incongruent conditions compared to the neutral conditions. Thus, the cortex demanded more oxygen to perform the incongruent - more difficult - tasks. Their study also sheds light on how the frontal lobe continues to develop into adulthood. Brain activation from Stroop interference increases from seven to about 29 years of age as the effect of this task decreases. While children and adults recruited neural processes from the left lateral prefrontal cortex, the response time for the adults was shorter, proving that maturation plays a statistically significant role in EF.

\subsubsection{Evidence for Development of Prefrontal Engagement in Memory}

In a study by Perlman, Huppert, and Luna (2015), the working memory aspect of EF was examined with fNIRS in children. The researchers worked with children between the ages of three to seven years old and their level of working memory maintenance on the lateral prefrontal cortex was assessed. A total of 68 children participated in the behavioral task called the monkey working memory task. Participants were asked to correctly identify the location of the monkey after it disappeared on a touch screen computer. The task was divided into two parts, long (six seconds) and short (two seconds) delay periods with four trials for both delay types. Behavioral results showed that age correlated with reaction time in both delay types. fNIRS results were acquired using the general linear model technique showing that the left frontal cortex and left dorsal prefrontal cortex activations were statistically significantly higher for the blocks requiring working memory engagement in 
contrast to the resting blocks. As for the different delay types, the extended delay period compared to the short delay showed higher activations in the right medial frontal and left lateral frontal cortices. The lateral prefrontal cortex showed more activation for spatial working memory than did the resting period. The results of this study correlate to similar findings, such that lateral prefrontal cortex activations can be seen in children as young as 3 years old and develop functionally to the age of 7 years regarding working memory but continue to build structurally up until adolescence.

\subsection{Justification for the current study}

This study is the first to examine the potential benefits of bilingualism on Hispanic bilingual preterm children in the United States, comparing their behavioral and neural results to monolingual preterm children. There is abundant evidence that prematurity significantly increases the risk for cognitive impairment in premature infants. While technological advances in the NICU helps clinicians to keep preterm-born children alive, we must also start asking, "how can we also help these infants to thrive?" While numerous studies have looked at the relative influence of clinical characteristics of the premature infant (i.e., respiratory distress, antenatal steroid use, infection, etc.) on cognitive development, no studies have ever examined the influence of language on their cognitive development. We know that language development is an important factor in a child's ultimate educational attainment (e.g., Head Zauche, Thul, Darcy Mahoney, \& Stapel-Wax, 2017). We also know that exposure to multiple languages may even potentiate or enrich development, specifically executive function (Kovacs et al. (2009) and Prior (2010)). Consequently, it is reasonable to suggest that bilingualism may in fact ameliorate the negative influence of prematurity on cognitive development. Bilingualism — being raised 
in two languages from birth—is known to enhance neurodevelopmental outcomes through the promotion of cognitive, and even physical, changes in the brain. It has yet to be determined if preterm born children that are bilingual have stronger EF skills than their preterm monolingual peers. To address this gap in the literature, the following research questions were examined:

\subsection{Research Questions}

1. Are there differences in executive functioning in monolingual versus bilingual pretermborn children, as measured by the DCCS task and the GNG task?

2. Are there differences in the neural recruitment of executive functioning in monolingual versus bilingual preterm children during the DCCS and GNG task, as measured by functional Near-Infrared Spectroscopy? 


\section{CHAPTER 3: METHODOLOGY}

The following sections elaborate on the processes that took place to answer the hypothesis presented in Chapter 1 and the research questions listed in Chapter 2.

\subsection{Participants}

A total of 16 right-handed children ( 9 bilinguals and 7 monolinguals), all between the ages of six to seven, participated in the study. All children were born preterm at Miami's Baptist Children's Hospital. An original pool of 22 children participated. Five children had to be eliminated from the study because of attention span and gross motor skill challenges due to cerebral palsy, $\mathrm{ADD}$, and $\mathrm{ADHD}$, or, because of excess noise during the tasks that rendered the NIRS data unreadable. Two children's data could not be used because of a data collection error: fNIRS recording stopped during the DCCS Task. This left the final participant pool with 16 preterm-born children. The study was approved by the Institutional Review Boards of both Florida International University and of Baptist Children's Hospital.

Determining children's language status was done via a parent questionnaire and brief interview with both the parent and the child. The language background questionnaire inquired about the child's language status, exposure, household income and SES background, reading activities done in English and in Spanish, and sources of language input. The monolingual children were easy to categorize. For the bilingual children, however, a strict operationalization of the construct was employed. Only children who actively used both English and Spanish, and critically, who answer in Spanish when addressed in Spanish by caregivers, were included in the bilingual group. Children who understood Spanish but do not produce in Spanish were not included in the study. That is, we were careful to only include children who had full productive capacity in Spanish. 
To confirm statistical comparability across groups, all children were administered the Picture Peabody Vocabulary Test (PPVT) in English. The bilingual group was also administered the Test de Vocabulario Imágenes Peabody (TVIP). These receptive vocabulary tests required the children to match the word given by the researcher with one of the four pictures presented on the monitor (English version) or the picture book (Spanish version). The vocabulary sets were categorized by age to determine the appropriate starting set. Their maximum capacity for the vocabulary set depended on the child making more than seven consecutive errors. Children were then equipped with the fNIRS cap and asked to perform two behavioral tasks measuring EF: the DCCS Task and Go/No-Go Task. There were no statistically significant differences among the monolingual and bilingual English vocabulary scores $(U=24.5, p=.470)$. This implied that all had excellent proficiency in English and could be administered the tasks in English, rendering them comparable for the study.

\subsection{Recruitment}

Parents gave written informed consent for their child to participate in the experiment, which was approved by the local International Review Board (IRB) prior to beginning the study. Children that were seven years of age also gave informed assent. Participants were recruited via flyers passed out at the annual Baptist prematurity event, and also, via social media. Additionally, only children that were right handed were recruited, since left-handed individuals tend to demonstrate significantly more lateralization when it comes to language (Szaflarski Rajagopal, Altaye, Byars, Jacola, 
Schmithorst, Schapiro, Plante, \& Holland, 2012).

\subsubsection{Birth factors}

Information on all the children's gestational age, birth weight, and length of stay in the NICU were also gathered at the onset of the study (see cohort data from Darcy Mahoney \& Baralt, 2016). The descriptive statistics for these variables are reported below in Table 1:

Table 1: Bio-info for Monolingual and Bilingual pre-term born children

\begin{tabular}{|l|l|l|l|}
\hline Group Means & $\begin{array}{l}\text { Gestational age } \\
\text { (weeks) }\end{array}$ & Birth Weight (g) & Length of Stay (days) \\
\hline Bilingual (n=9) & 29.9 & 1450 & 57.9 \\
\hline Monolingual (n=7) & 31.2 & 1517 & 47.7 \\
\hline
\end{tabular}

There were no statistical differences among the monolingual versus bilingual preterm-born children in any of these variables. However, notably, the bilingual group was on average born earlier than the monolingual group, weighed less, and was in the NICU for more time (an average of ten days more).

\subsection{Behavioral Tasks}

\section{DCCS}

For this study, the Dimensional Change Card Sort Task (Doebel \& Zelazo, 2015; Morigucho \& hiraki, 2013; Zelazo, 2006) was first piloted with five children. Specifically, this task tests an individual's selective attention skills, memory, and cognitive shifting. Following Zelazo (2006), the participant was shown a background board with three cards: 
a blue rabbit, a blank card, and a red boat. These were all placed at eye level (Figure 1). To assist in sorting the cards, children were also equipped with a rubber finger for grip. The participant was instructed to draw a card from the dispenser and match it to the permanent cards according to the given instructions. Three sets of instructions were given to the participant for the three different games: color, shape, and border.

The color game consisted of the participant matching the color of the card drawn with the color of the card presented on the board. For example, irrespective of shape, the blue boats had to be matched to the blue rabbit. The shape game consisted of the participant matching the shape of the card drawn with the card on the board. For example, irrespective of color, the boats had to be matched to boats and rabbits to rabbits. The border game consisted of the participant playing the color game if the card drawn had a black border and playing the shape game if there was no border. This game was the most difficult, as the participant had to play both games at the same time and remember that the presence of a black border meant to play the color game.

These games tested the children's ability to remember the instructions, and their ability to switch between instructions. For the blank card (whose purpose was to obtain baseline data for fNIRS), children simply had to sort blank cards from the middle dispenser and sort them into the middle tray. During the entire task, a researcher was sitting next to the child and explained the instructions. The child practiced each trial type before beginning. The task only began once the child was able to verbalize in his/her own words how to play all three games. Data from this task is reaction time and accuracy for all three trial types. 

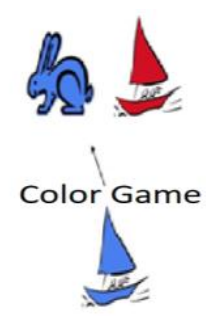
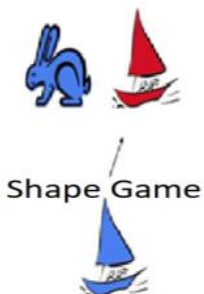

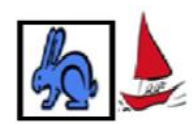

Border Game

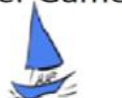

Figure 1: Dimensional Change Card Sort Task Schematic with Bunnies and Boats

GNG

The second behavioral task given was the Go-No-Go (GNG) task (Fishburn et al., 2019). This task was also piloted with the same five children, in which there are two trials: Go, in which a tiger and elephant appear on the monitor screen interchangeably, and NoGo, in which a tiger, elephant, and giraffe appear interchangeably. For each event, the participant was instructed to press the button provided as soon as the tiger or elephant appeared, but not for the giraffe. In the go trial, the child had to press the button for each animal. In the no-go trial however, the giraffe appeared randomly, and so the task was to

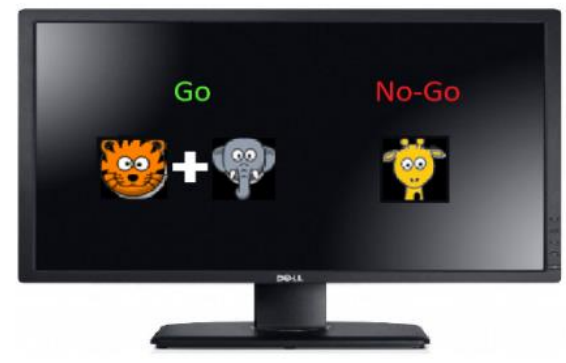

Figure 2: Go No-Go setup to test response inhibition

not press the button for this item type. This task specifically tests the child's inhibitory skills, exercising their ability to suppress responses to certain cues, and memory, exercising the ability to retain and then perform multiple instructions at once (Nosarti et al., 2007; Orchinik, 2014). Data from this task is reaction time and accuracy for both trial types. 


\subsubsection{Button for Go/No-Go Task}

Designing and creating a button that could be connected to both the task and to NIRS took substantial time and piloting. The goal was to create a button that would generate automated reaction times and score the accuracy of the recorded data. Several versions were piloted and tested with the pilot group children; we also asked these children for their opinion. In the end, the final button was a large green button placed on an incline to make the child's arm and hand as comfortable as possible. The button used for this task was created and designed for this experiment by Mr. Anil Thota, head biomedical engineer in the ANS lab at Florida International University.

\section{5 fNIRS}

The NIRx fNIRS imaging system includes multiple features for recording neural signals. All data were collected on the NIRStar15-2/Homer 2. Features involved were a near infrared spectroscopy (NIRS) child's-sized headband to place on the head covering the frontal lobe area, NIRStim 4.0 (for data curation; making temporal blocks), and an 8 emitter and 8 detector optode system (please see Figures 3). The probes on the cap are spaced about .15 centimeters apart in a 10-20 coordinate system that provides coverage of the frontal lobe. The DCCS task was synched with fNIRS recordings by creating a temporal block system, in which the duration of the Control (blank), Color, Shape, and Border trials were automated (see section 3.6 below), sounding a beep indicating the start and end of a trial. Thus, these blocks act as markers and create a visual representation of the neural activity in correspondence to its respective trial. Similarly, the GNG task was paired with fNIRS employing the block system, like the set up for the DCCS. 


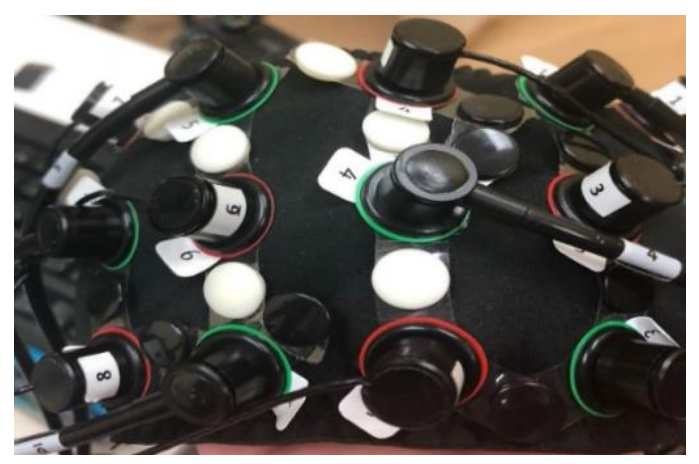

Figure 3: fNIRS Prefrotnal Lobe Headband containing 8 emitters (green) and 7 detectors (red)

The placements of the probes and the interaction between the emitters/detectors that were used for this study are shown in Figure 5. Additionally, the Broadman areas listed theoretically correlate to specific features of executive function skills, which include memory, cognitive flexibility, and attention:

\subsection{Procedure}

After children completed the questionnaires and Peabody vocabulary tests, the fNIRS cap was placed on their head. To ensure correct placement, a picture was taken to maintain uniformity among the participants. Headband placement took time because it was critical to ensure direct scalp contact with each emitter and detector; all hair had to be pushed out of the way. Once the participant gave verbal confirmation of comfort, the fNIRS system was calibrated to confirm that the detectors and emitters were receiving a clear signal. Three colors are used by the NIRStar 15-2 system to differentiate between a good and bad signal according to the coordinate system in relation to the cap. Green represents an "excellent" signal, yellow is "acceptable", red is "critical", and white is a "lost" signal. If there were three or more red boxes, then the cap required adjusting for hair blocking those specific probes. This was done by gently moving aside the hair using a long cotton 
swab; at times, we had to completely start over to try and get a better fitting and contact. When the calibration was complete, one of the behavioral tasks (DCCS or GNG) was loaded into the NIRStim 4.0 system (note: the order of tasks was randomized for participants). Each participants' calibrations were recorded and saved for later analysis. After set-up, calibration, and initial data was saved, the participant was given the instructions and the behavioral task began. All children's tasks were video-recorded. The fNIRS portion of the experiment lasted about 20-30 min per participant if there were no set up complications. Once both tasks were completed, the cap was removed. For their time and participation, families were compensated a $\$ 100$ visa gift card. The children also received a prize from the prize box.

\subsection{Coding and Analysis}

\subsubsection{DCCS Task}

Reaction time (RT) and accuracy were recorded for each participant and from each task. Following the same methodology of Moriguchi and Hiraki (2013), RT for the DCCS task was determined manually by retrospectively watching the video recording of the child's task performance. This was done by obtaining the milliseconds from the FPS $(1 / 30)$ of the video recordings via VLC Media Player. A protocol was created to ensure all participants were scored the same to reduce error. RT was based on two markers: 1) time the card left the dispenser 2) time the card left the child's hand. Accuracy for the DCCS task was determined by scoring the number of correct responses for the color, the shape, and the border trials separately. A non-parametric test, the Mann-Whitney U test, was used to statistically compare the groups since the data was not evenly distributed. 


\subsubsection{Go/No-Go Task}

RT for the Go/No-Go task was automated through the block system that was set up in the NIRS software to determine the time it took the child to press the button once the image appeared. A MATLAB code was created to extract RT for each participant, coding for the time of the response minus the time of the stimulus appearing on the screen. Accuracy for the Go/No-Go task was determined based on whether or not participants correctly pressed the button for the appropriate stimuli, in comparison to the exact timing in which the stimuli appeared. As with the DCCS task, the Mann-Whitney U test was used to statistically compare groups' RT and accuracy.

\subsection{3 fNIRS}

The NIRStar data acquisition was converted to Homer2 data format and inserted into the NIRS toolbox. The NIRStim 4.0 was used to create temporal blocks for the DCCS and GNG tasks. The DCCS was broken into events and conditions called Control, Color, Shape and Border, which were all 20 seconds each. The neural activity in these blocks were evaluated by setting markers to show which blocks had increased or decreased activity. The GNG was broken into two baselines (24s), six Go_Beeps (.5s), and six No_Beep (.5s), with the transitions (.2s) in between. NIRStar 15-2 was used to calibrate, preview, and record the signals received from the probes attached to the NIRS cap.

To help prevent any external artifacts, a protocol was set in place to reduce physical movement. A Velcro strap and "best" position were established, to minimize head and cable movements during the task, in addition to verbally communicating to the participants to try their hardest not move any parts of their body except for their hands needed to complete the tasks: drawing the cards from the deck for the DCCS task, and pushing the 
button for the GNG task. The recorded neural activity for each participant was saved by date of the experiment and the participant number for later analyses. Once all neural recordings were collected, they were saved in separate folders organized by language group and behavioral task (i.e. DCCS and GNG). The NIRSlab 2017.6 was used to load the experimental raw data via the imager system NIRScout/NIRSport, to create event markers, and preprocess the data by trimming the data before and after event blocks and check data quality. These steps were critical in order to remove excess noise.

The following analysis was completed via NIRS toolbox. First, the data was converted from voltage (V) to optical density (OD). Second, a 4th order Butterworth band pass filter was applied to remove any physiological noises within $.01-0.5 \mathrm{~Hz}$, and then converted OD to oxy- hemoglobin and deoxy-hemoglobin via Modified Beer Lambert Law with a partial pathlength factor of 5/50 (Jacques, 2013) at a sampling rate of $7.81 \mathrm{~Hz}$. Next, and to remove any motion artifacts, which typical arose from sharp movements of the head and/or speaking, a Temporal Derivative Distribution Repair (TDDR) was applied to downweigh outlier fluctuations (Fishburn et al., 2019). In order to check the integrity of the data for all subjects, a MATLAB routine was created.

To analyze the data, a Statistical Parametric Mapping was performed on subject and group level. The data was first fitted using the Generalized Linear Model (GLM) - a linear equation system that mimics the response data.

$$
\boldsymbol{Y}=\boldsymbol{X} * \boldsymbol{\beta}+\boldsymbol{\varepsilon}
$$

The equation above represents the GLM. Y represents the response signal that was collected per participant. $\mathrm{X}$ represents the design matrix, which includes boxcar functions (i.e temporal blocks) convolved with the canonical hemodynamic response model 


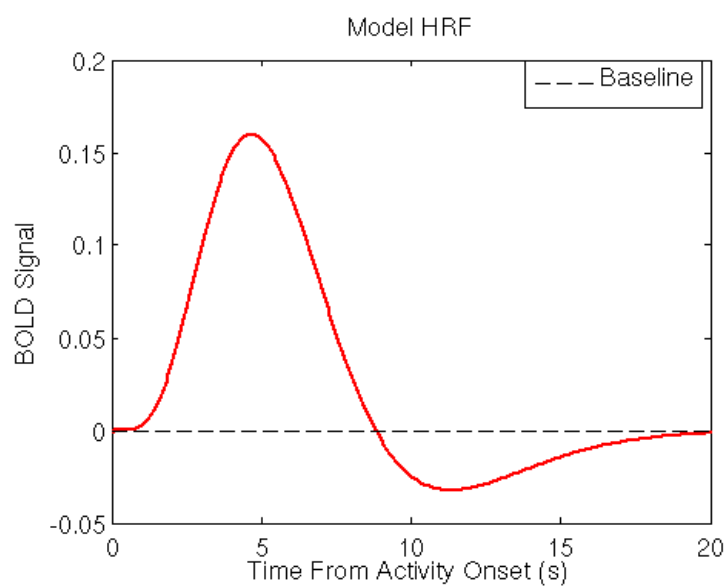

Figure 4: Model of Hemodynamic Response Function (HRF) for a Blood-Oxygen-Level-Dependent (BOLD) signal

producing the HRF model seen below in Figure 4 for each condition. $\beta$ (beta) represents the estimated coefficients, which were calculated via autoregressive iteratively-reweighted least squares method.

The next step was to account for the slow drift that is seen in a BOLD response by applying the 3rd -order Legendre polynomial repressor (Barker, Aarabi, \& Huppert, 2013). Finally, the white noise is represented by $\varepsilon$. The Design matrix and fNIRS data was inputted into the GLM model to estimate subject level stats. Subject level t-test contrasted were performed to determine the effects of each task condition per participant. A Group level statistical analysis was then performed using a linear mixed effects model fit via maximum likelihood method. The effects of the conditions for each group, was controlled for subject on $\mathrm{HbO}$ and $\mathrm{HbR}$ concentration levels across subjects within each language group. To control for the interdependence of measurements (e.g., artifacts or physiology that share noise across multiple channels) by using the full covariance noise model. 
A

\begin{tabular}{|c|c|c|}
\hline $\begin{array}{c}\text { fNIRS } \\
\text { Channel }\end{array}$ & Source & Detector \\
\hline 1 & 1 & 1 \\
\hline 2 & 1 & 2 \\
\hline 3 & 2 & 1 \\
\hline 4 & 2 & 3 \\
\hline 5 & 3 & 1 \\
\hline 6 & 3 & 2 \\
\hline 7 & 3 & 3 \\
\hline 8 & 3 & 2 \\
\hline 9 & 4 & 2 \\
\hline 10 & 4 & 4 \\
\hline 11 & 4 & 5 \\
\hline 12 & 3 & 5 \\
\hline 13 & 5 & 4 \\
\hline 14 & 5 & 8 \\
\hline 15 & 6 & 4 \\
\hline 16 & 6 & 5 \\
\hline 17 & 6 & 8 \\
\hline 18 & 6 & 7 \\
\hline 19 & 7 & 5 \\
\hline 20 & 7 & 7 \\
\hline 21 & 8 & 8 \\
\hline 22 & 8 & 7 \\
\hline
\end{tabular}

B

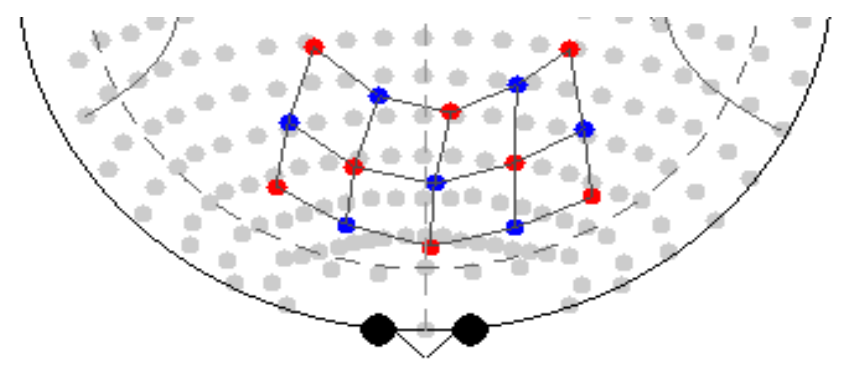

$\mathbf{C}$

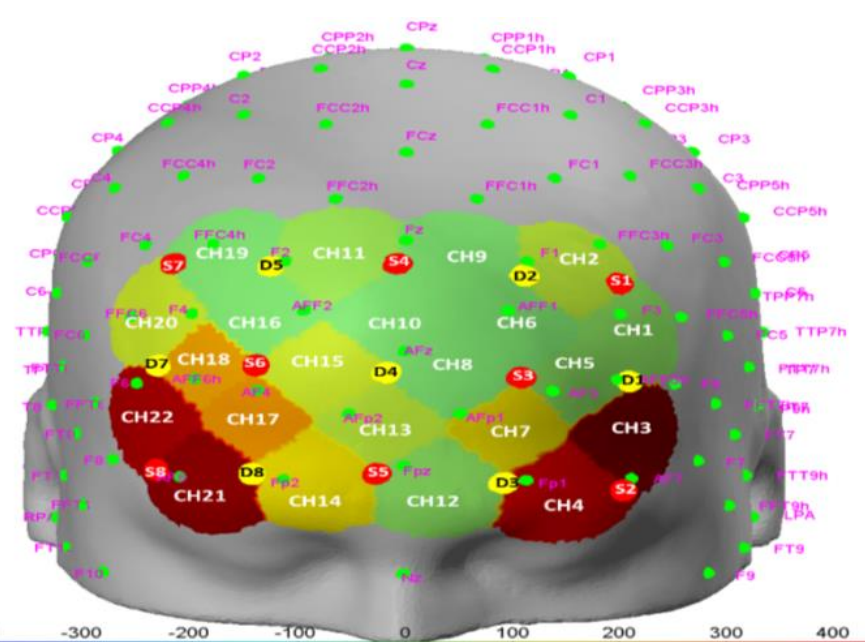

\begin{tabular}{|c|c|c|c|}
\hline $\begin{array}{l}\text { EEG } \\
\text { Electrode }\end{array}$ & $\begin{array}{l}\text { fNIRS } \\
\text { Channel }\end{array}$ & $\begin{array}{l}\text { Broadmann } \\
\text { Area }\end{array}$ & Area Involved In \\
\hline $\begin{array}{l}\text { FP1, AFp1, } \\
\text { FPz } \\
\text { Fp2, AFp2 }\end{array}$ & $4,7,12$ & $10 \mathrm{R}$ & $\begin{array}{l}\text { Executive function } \\
\text { Memory Encoding and } \\
\text { retrieval } \\
\text { Use of Language } \\
\text { (generating sentences, } \\
\text { word-stem completion } \\
\text { verbal fluency, and syntactic } \\
\text { processing) }\end{array}$ \\
\hline $\begin{array}{l}\text { AF7 } \\
\text { F8, F6 }\end{array}$ & $\begin{array}{c}3 \\
21,22\end{array}$ & $\begin{array}{l}46 \mathrm{~L} \\
46 \mathrm{R}\end{array}$ & $\begin{array}{l}\text { Working memory } \\
\text { Sustaining attention } \\
\text { Regulating self-control }\end{array}$ \\
\hline $\begin{array}{l}\text { AF3, Afz } \\
\text { Afz, AF4 }\end{array}$ & $\begin{array}{l}5,8,10 \\
17,15\end{array}$ & $\begin{array}{l}9 \mathrm{~L} \\
9 \mathrm{R}\end{array}$ & $\begin{array}{l}\text { Motor planning } \\
\text { Organization } \\
\text { Regulation }\end{array}$ \\
\hline $\begin{array}{c}\text { F4, F2, AFF2 } \\
\text { Fz, F1, F3 }\end{array}$ & $\begin{array}{c}20,11 \\
16 \\
9,2,1\end{array}$ & $\begin{array}{l}8 \mathrm{R} \\
8 \mathrm{~L}\end{array}$ & $\begin{array}{l}\text { Management of uncertainty } \\
\text { Control of eye movement }\end{array}$ \\
\hline
\end{tabular}

Figure 5: Electrode Placement and fNIRS Channel connections. A) The fNIRS emitter and detector interactions are displayed. B) Montage gives a visual of the areas of significant activation. C) Table with Broadmann Areas that correlate to the probe arrangements. 


\section{CHAPTER 4: RESULTS}

The purpose of this study was to examine the potential for bilingualism to enhance the executive function skills of preterm-born children as compared to monolingual pretermborn children. The study did this by examining the outcomes of both the behavioral tasks (DCCS task, Go/No-Go task) and the brain imaging data via fNIRS. The results below address the two research questions previously formulated to test the hypothesis that raising children who are born pre-term as bilinguals may have a positive influence on their neuroplasticity process and enhance their executive functioning skills, bridging the gap for low EF performance. Each research question is answered in turn.

\subsection{Behavioral task outcomes}

The first research question asked whether there were differences in executive functioning in monolingual versus bilingual preterm-born children, as measured by the DCCS task and the GNG task.

\subsubsection{DCCS}

A quick visual inspection of children's mean reaction time data (provided in Table 1 below) show that across the three trial types of the task, there appear to be no major differences of scores in accuracy between the bilingual and monolingual participants. Contrarily, there did appear to be a difference in the groups' reaction times. The bilingual preterm-born children averaged just over six seconds on the three trials, while monolingual preterm-born children averaged over 11 seconds. These results are shown in Figure 6 and 7. 


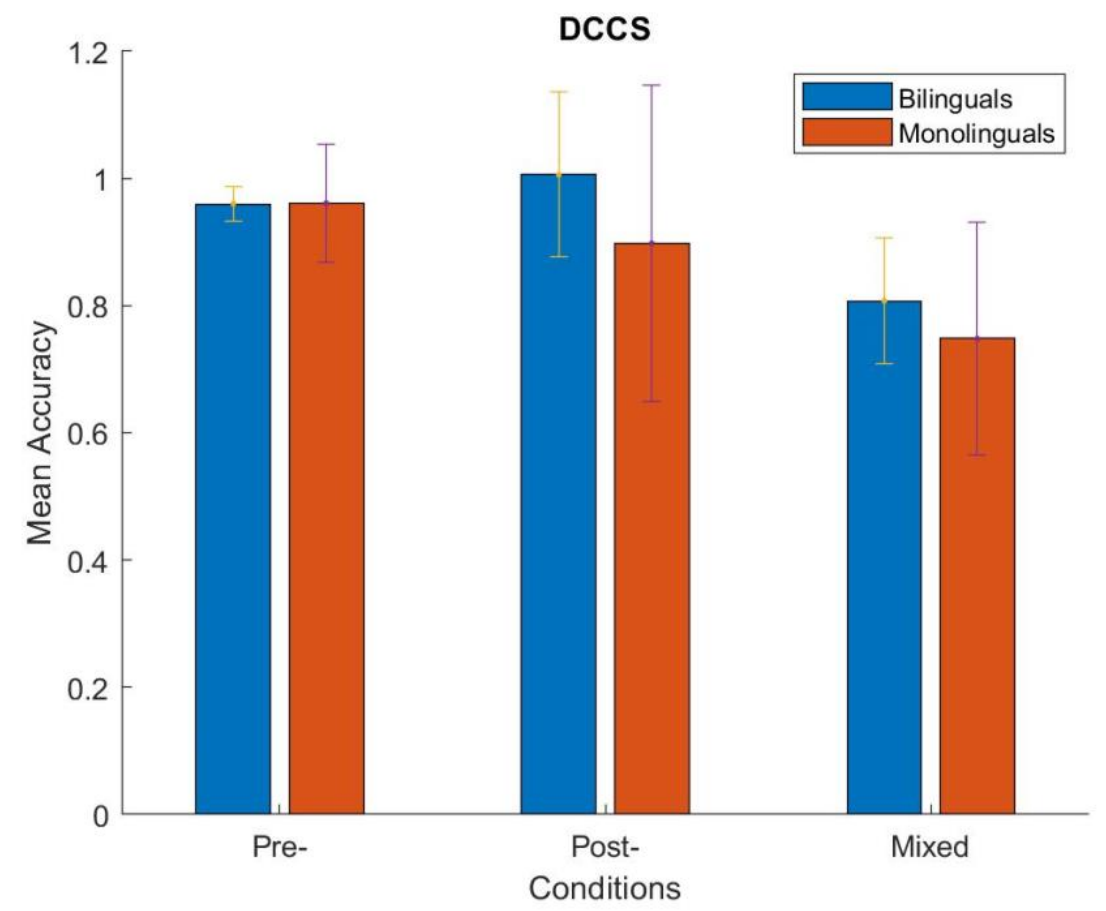

Figure 6: DCCS Accuracy for Bilinguals vs. Monolinguals. Pre-trial $(p=.918)$, Post-trail $(p=.918)$, Mixedtrial $(p=1.0)$

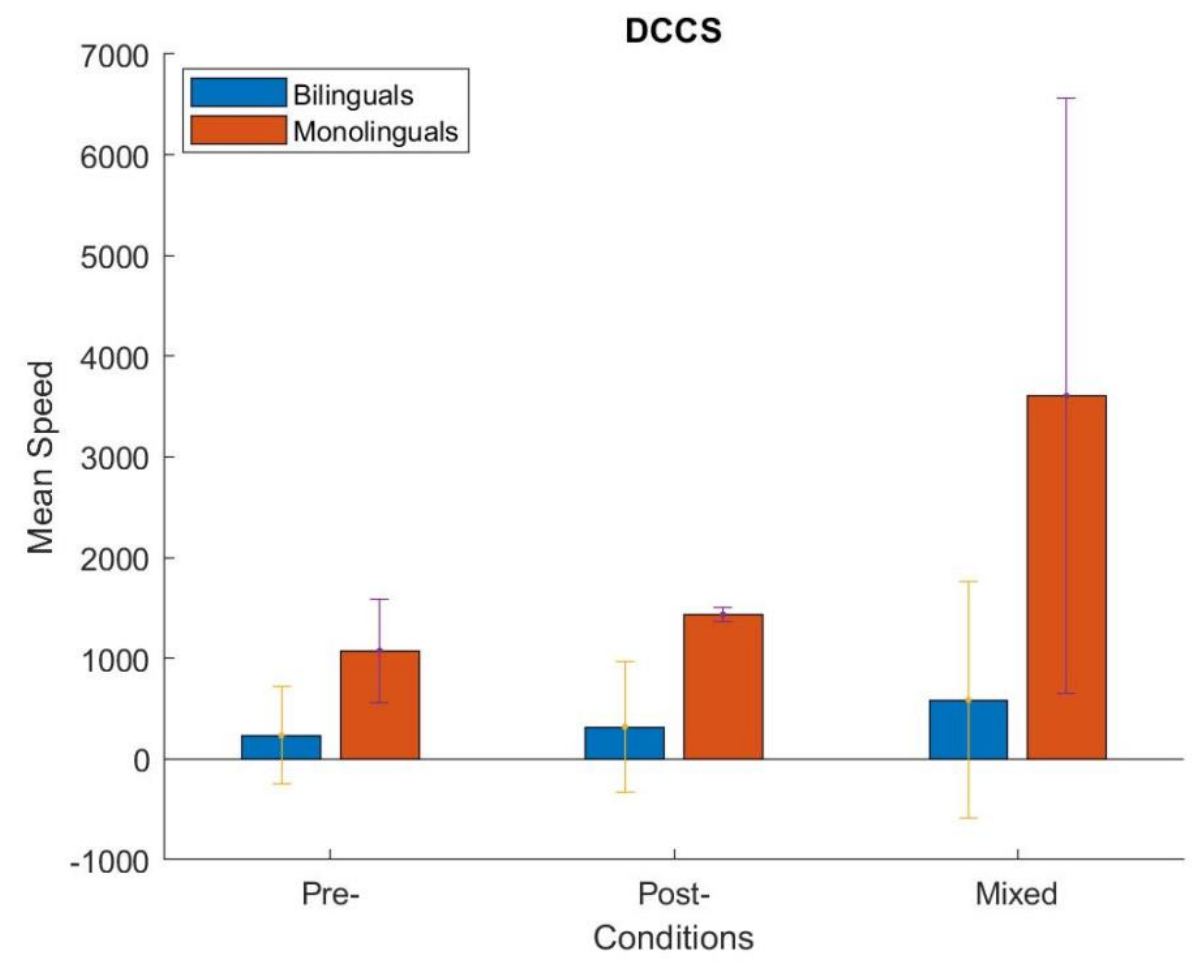

Figure 7: DCCS Reaction Time for Bilinguals vs. Monolinguals. Pre-trial $(p=.016)$, Post-trail $(p=.016)$, Mixed-trial $(p=.023)$. 
Below is the descriptive statistics for both groups showing the accuracy and reaction times for the Dimensional Change Card Sort Task. Shown in bold are the standard deviations for all task conditions that were significant.

Table 1: Descriptive Statistic for DCCS Task Performance

\begin{tabular}{|c|c|c|c|c|c|}
\hline Accuracy/Reaction & $\mathbf{N}$ & Mean & Std. & Minimum & Maximum \\
\hline Correct PreTrial & 16 & .9605 & .08511 & .75 & 1.00 \\
\hline Correct PostTrial & 16 & .9587 & .32918 & .34 & 1.94 \\
Correct Border & 16 & .7741 & .24659 & .25 & 1.00 \\
\hline RT Pretrial & 16 & 602.92 & $\mathbf{6 4 6 . 5 3}$ & .00 & 1503.00 \\
\hline RT Post & 16 & 808.13 & $\mathbf{8 9 0 . 2 2}$ & .00 & 2125.06 \\
\hline RT Border & 16 & 1909.35 & $\mathbf{2 5 7 2 . 4 8}$ & .00 & 8848.75 \\
\hline
\end{tabular}

\subsubsection{Statistical analysis, DCCS task}

For the DCCS task, a Mann-Whitney U test was run to statistically compare the differences between both groups. No statistical difference was found between groups on the pre-trial $(U=30.0, p=.918)$, post-trail $(U=30.0, p=.918)$, or mixed-trial $(U=31.0$, $\mathrm{p}=1.0)$ for accuracy. However, results confirmed that the differences in reaction times were statistically significant, for the pre-trial $(U=9.50, p=.016)$, post-trail $(U=9.50, p=$ $.016)$, or mixed-trial $(\mathrm{U}=10.5, \mathrm{p}=.023)$. This implied that while all children performed about the same in terms of accuracy, the bilingual children were statistically significantly faster in their performance on the DCCS task on all three trials. 


\subsubsection{GNG}

Descriptive data from the Go/No-Go task showed a similar pattern to the DCCS task. In terms of accuracy, the monolingual and bilingual preterm-born children's accuracy were similar. It appeared that there was no statistically significant difference between the two groups. Reaction time, however, demonstrated a statistical difference. Figures 8 and 9 below illustrate the Go/No-Go task accuracy and reaction times respectively for bilingual vs. monolinguals.

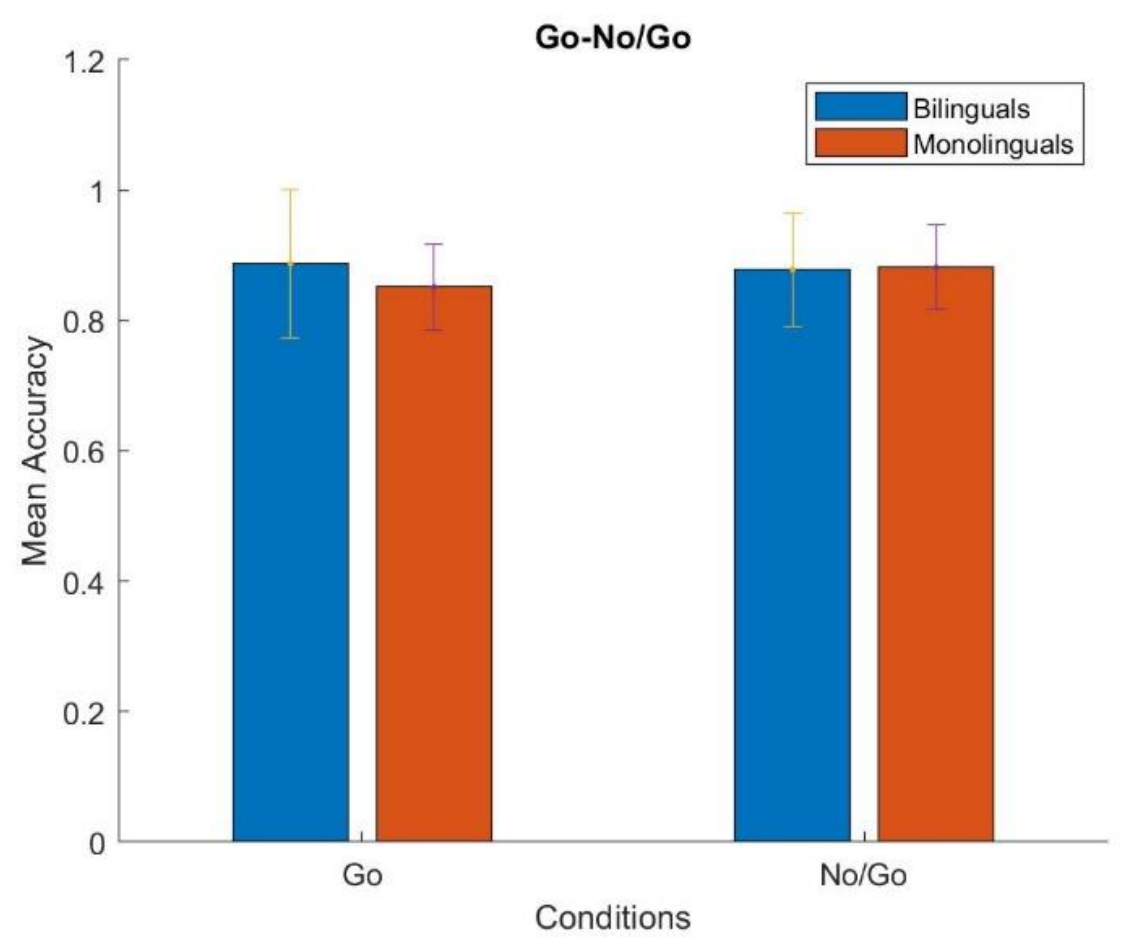

Figure 8: GNG Accuracy for Bilinguals vs. Monolinguals. Go trial $(\mathrm{p}=.352)$ and No-Go trial $(\mathrm{p}=.476)$ 


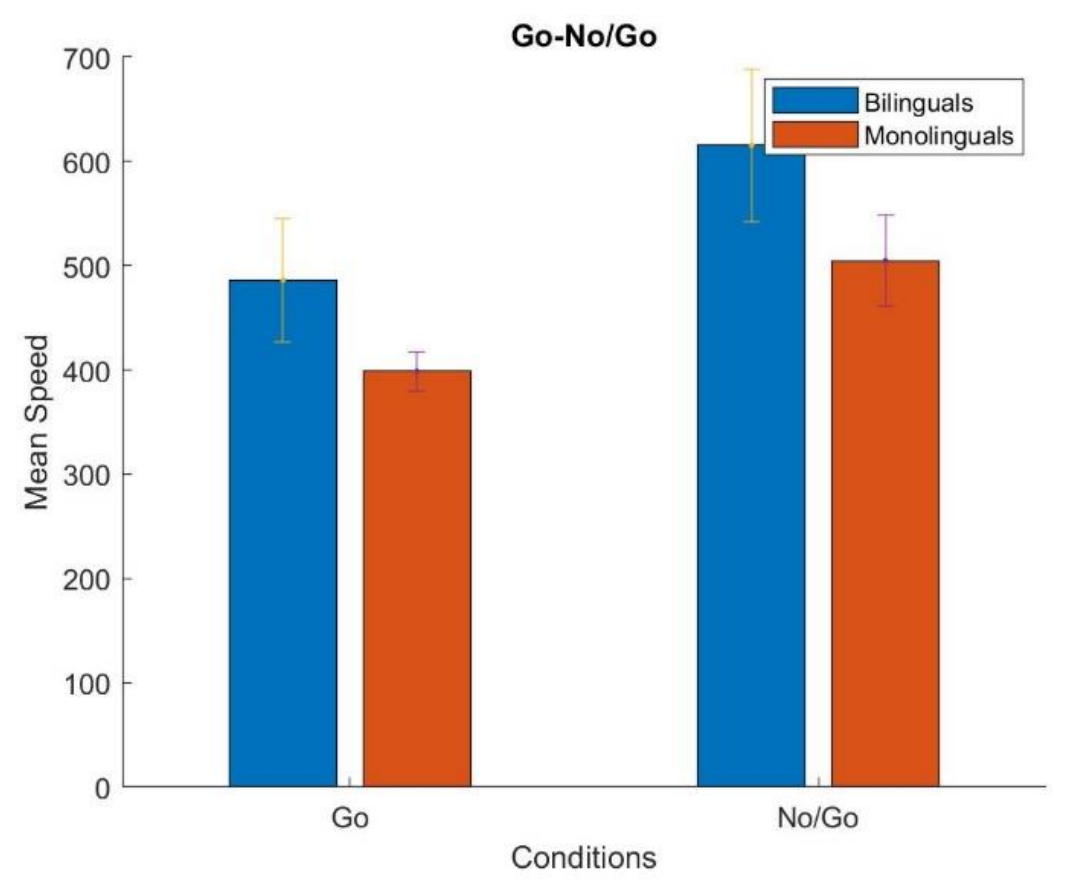

Figure 9: GNG Reaction Time for Bilinguals vs. Monolinguals. Go trial $(\mathrm{U}=3.0, \mathrm{p}=.057)$ and No-Go trial $(\mathrm{U}=3.0, \mathrm{p}=.057)$

Below is the Descriptive statistics for both groups showing the accuracy and reaction times for the Dimensional Change Card Sort Task. Shown in bold are the standard deviations for all task conditions that were significant.

Table 2: Go/No-Go Task

\begin{tabular}{|c|c|c|c|c|c|}
\hline $\begin{array}{c}\text { Accuracy/Reaction } \\
\text { Time }\end{array}$ & $\mathbf{N}$ & Minimum & Mean & $\begin{array}{c}\text { Std. } \\
\text { Deviation }\end{array}$ & Maximum \\
\hline Correct Go Trials & 10 & .69 & .8731 & .09481 & .98 \\
\hline Correct No Go Trials & 10 & .74 & .8796 & .07475 & .96 \\
\hline RT Go Trials & 10 & 376.24 & 451.16 & $\mathbf{6 4 . 1 8}$ & 554.96 \\
\hline RT No Go Trials & 10 & 440.15 & 571.07 & $\mathbf{8 2 . 8 2}$ & 718.23 \\
\hline
\end{tabular}




\subsubsection{Statistical analysis, Go/No-Go task.}

Statistical analyses confirmed that these differences in reaction times for both tasks were statistically significant. For the GNG task, a Mann-Whitney U test was run to statistically compare the differences between both groups. No statistical difference was found between groups on the Go trial $(U=7.0, p=.352)$ or No-Go trial $(U=8.5, p=.476)$ for accuracy. However, the test showed that there were statistical differences in their reaction time. This implied that while all children performed about the same in terms of accuracy, the bilingual children were again statistically significantly faster in their performance on the GNG task for Go trial $(U=3.0, p=.057)$ and No-Go trial $(U=3.0, p$ $=.057)$.

\subsubsection{Summary for Research Question 1}

In sum, the monolingual and bilingual preterm-born children performed similarly on both tasks in terms of accuracy. There were no statistical differences in their performance on both the DCCS or Go/No-Go task. In terms of reaction time, however, the bilingual preterm-born children statistically significantly outperformed the monolingual preterm-born children on both tasks.

\subsection{Neural outcomes}

The second research question asked whether there were differences in the neural recruitment of executive functioning skills in monolingual versus bilingual preterm 
children during the the DCCS and GNG task, as measured by fNIRS.

\subsection{1 fNIRS results for DCCS task}

The statistically significant areas of activation are shown in the following figures below. The SPM t-statistic outputs for a single participant and for each language group show the oxy- and deoxy-hemoglobin differences per condition and mixed conditions (contrasts). Some of the results were obtained by the making contrasts by subtracting the rest condition from the task condition (e.g. Shape-Control). The interpretation for both oxyhemoglobin and deoxy-hemoglobin are the same, but they have opposite meanings. To interpret these outputs, first the red and blue areas must be understood. For oxyhemoglobin, red areas signify an increase of oxygenated hemoglobin to those specific fNIRS channels, while blue represents a decrease. On the other hand, for deoxyhemoglobin, red areas signify an increase of deoxygenated hemoglobin to those specific

fNIRS channels, while blue represents a decrease. It is important to note that blue areas do not represent deoxy-hemoglobin only.

4.2.1.1 Analysis of a Single participant with oxy-hemoglobin (hbo) and deoxyhemoglobin (hbr)

Individual data is shown below in Figure 10. In Figure 10 the SPM t-image for a monolingual participant presented for each condition of the DCCS Task. The shaded areas signify where t-statistic was greater than the threshold (i.e. $\alpha=.05$ ). 


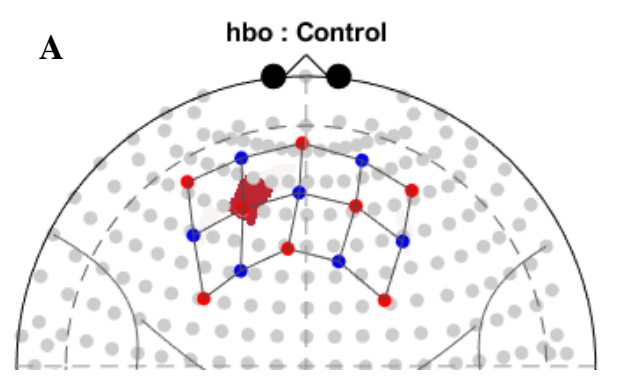

$\mathbf{E}$
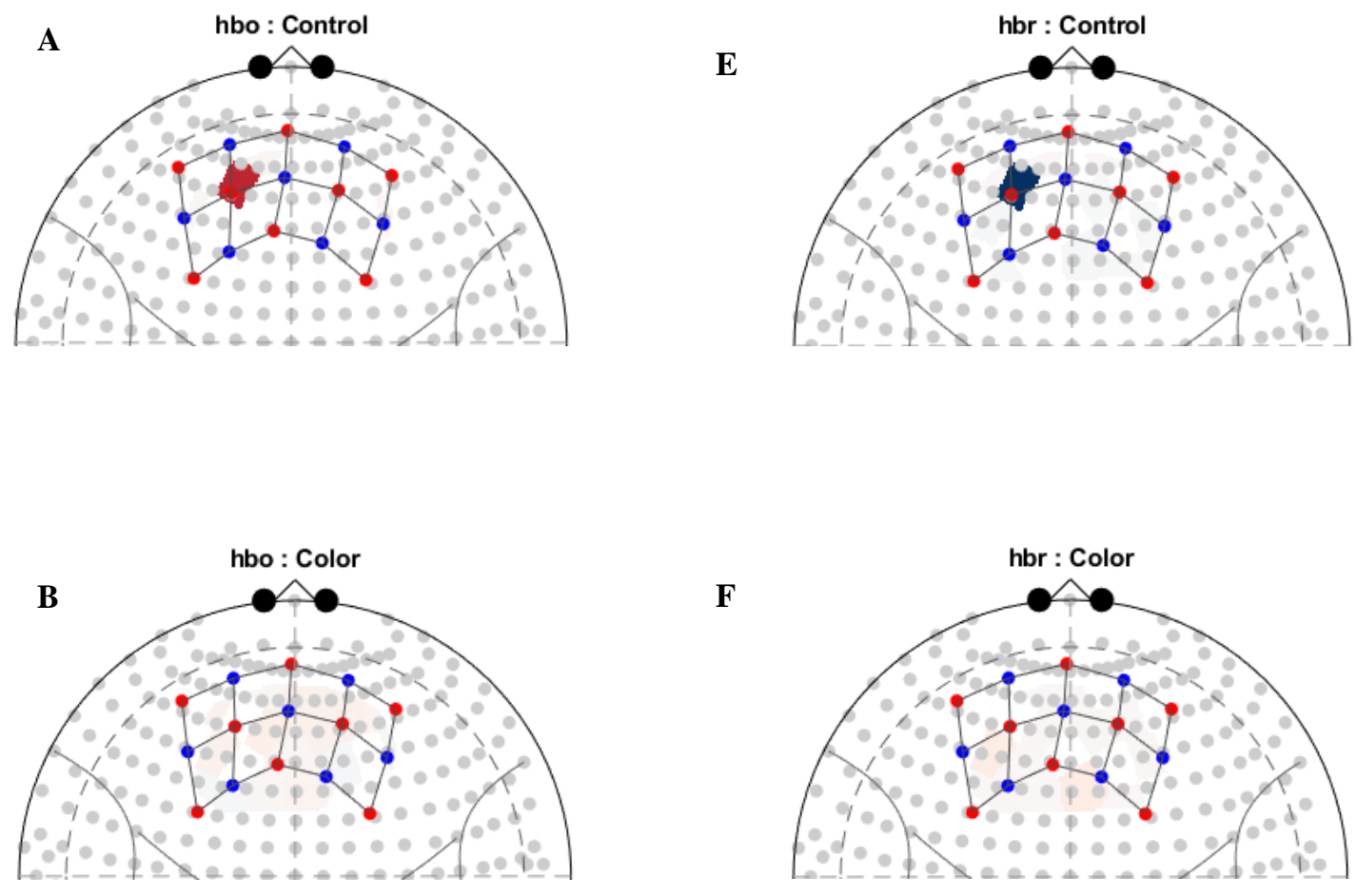

$\mathbf{F}$

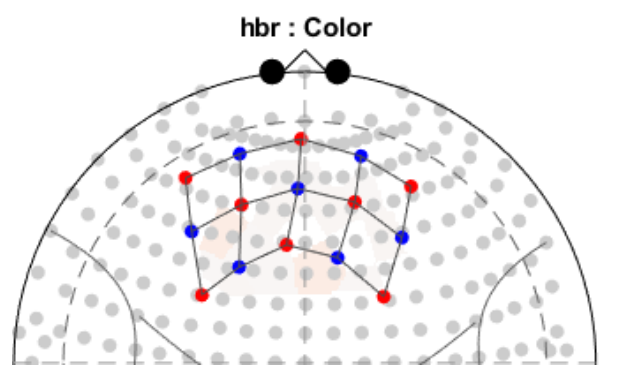

2

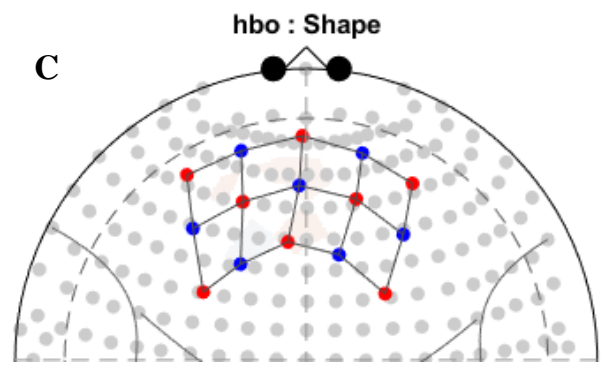

G
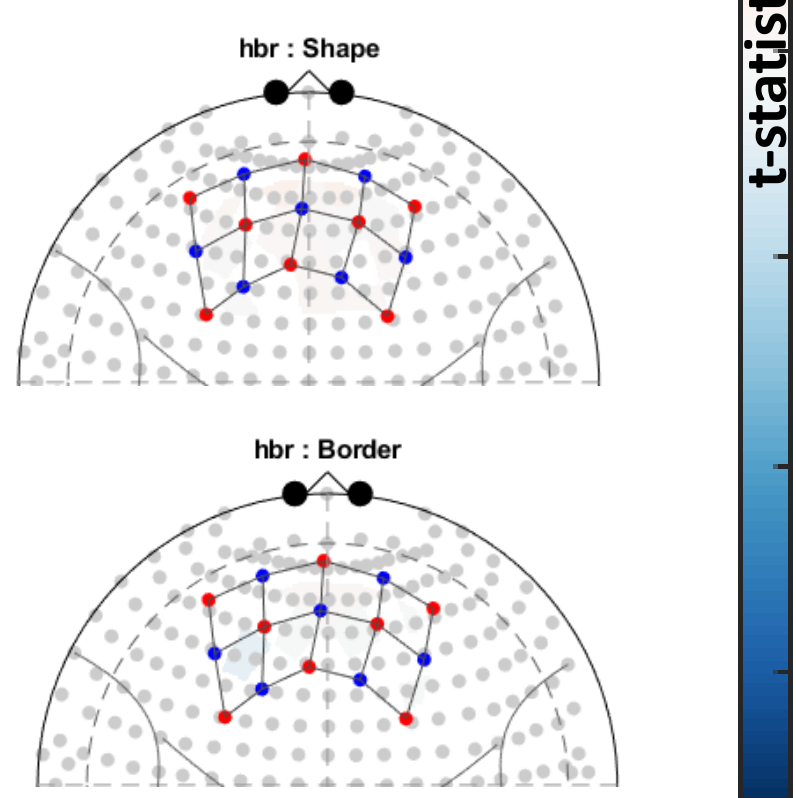

H
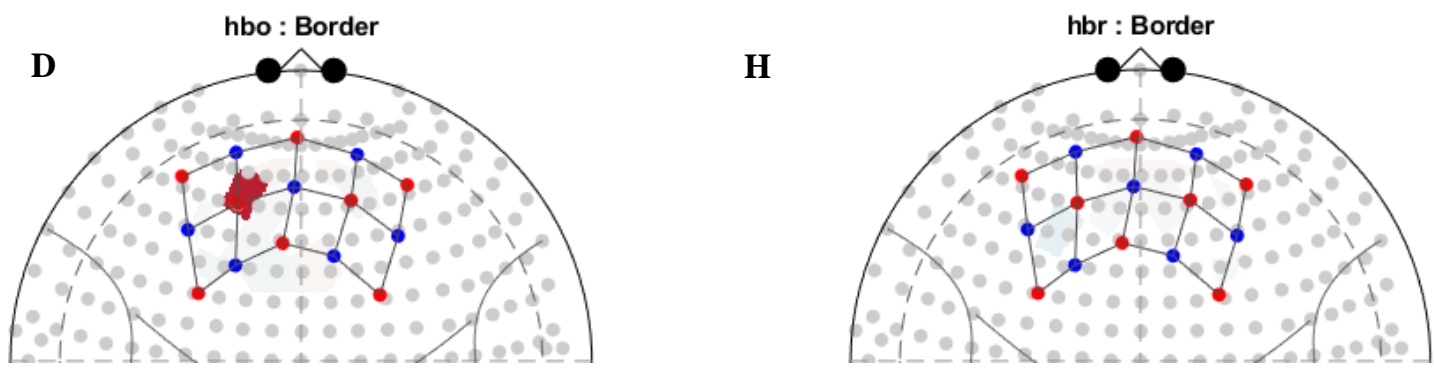

Figure 10: SPM T-map simulated via NIRS Toolbox. t-map of oxy-/deoxy-hemoglobin activations (all channels) for single monolingual participant.

To illustrate this, let us look at the fNIRS images generated for participant 12 during the DCCS task. As can be seen in Figure 10, this monolingual participant is showing activation roughly in the center of channels 5, 6, 7, and 8 for both Control (blank card trial) 
and Border Conditions in the oxy-hemoglobin state. The Control Condition for the deoxyhemoglobin state has the same area of activation as the oxy-hemoglobin; however, this area is blue. This signifies that the participant had a decrease in deoxy-hemoglobin because of the increase in oxy-hemoglobin. The red statistically significant areas - positive t-values and blue areas - negative t-values - represent statistically significant differences between the fitted General Linear Model and the Hemodynamic Response Function (HRF) convolution. This serves to demonstrate that these areas of the frontal lobe represent hemoglobin concentration changes, which are highlighted by the blue and red areas. These are responsible for performing the task for this specific individual.

\subsubsection{Analysis of each Language Group}

Group data is shown below in Figure 11. In Figure 11, the SPM t-image per language group for each condition of the DCCS Task is provided: 


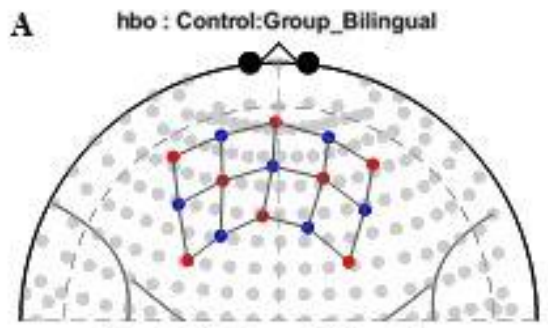

hbo: Color:Group_Bilingual

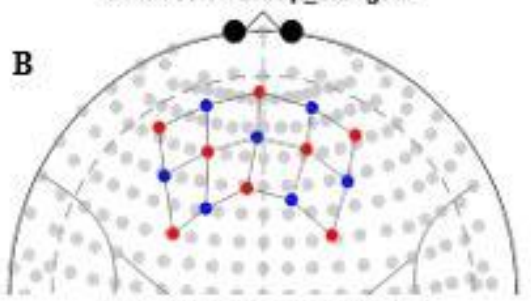

hbo: Shape:Group_Bilingual

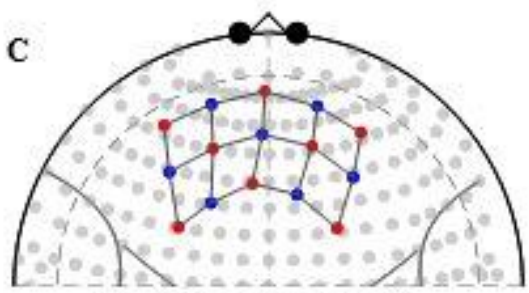

hbo: Border:Group_Bilingual

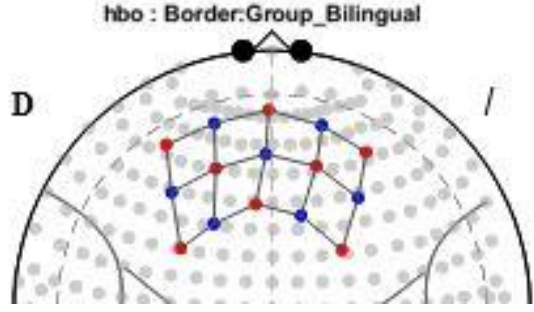

E hbo: Control:Group_Monolingual

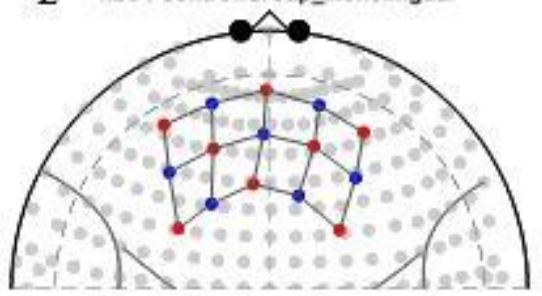

hbo : Color:Group_Monolingual

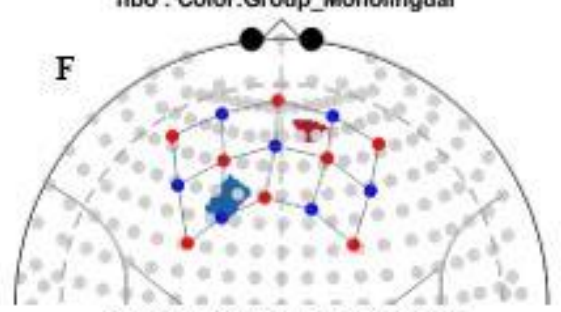

hbo : Shape:Group_Monolingual

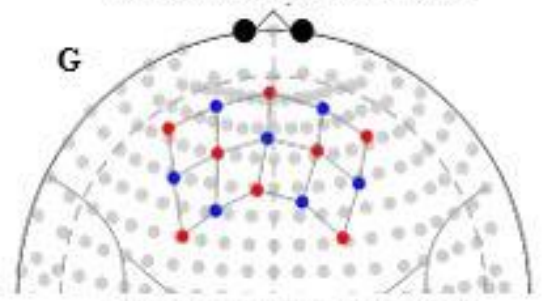

hbo : Border:Group_Monolingual

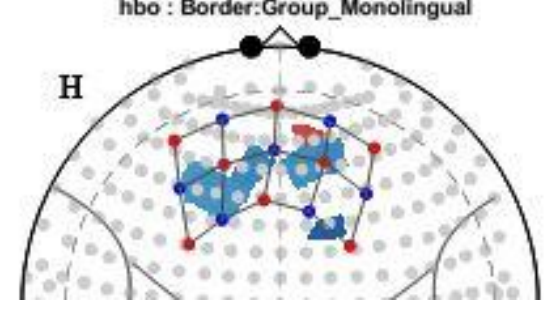

Figure 11: SPM T-map simulated via NIRS Toolbox. $t$-map of oxy-hemoglobin averaged channels per language group.

As can be seen, both similarities as well as differences exist between the two groups. The similarities were noticed between the groups for the Control and Shape Conditions. The differences were in the Color and Border Conditions, where the monolinguals had statistically significant positive t-values in Channels 14 (11A and 11F). This implies that the monolinguals recruited from different areas and/or recruited more oxy-hemoglobin than bilinguals, showing their need for an increase of oxygenated hemoglobin to those specific channels to perform the given task. 
It is also informative to consider the deoxygenated levels as well. In Figure 12 below, the deoxy-hemoglobin SPM per language group for each condition of the DCCS Task are shown:

A

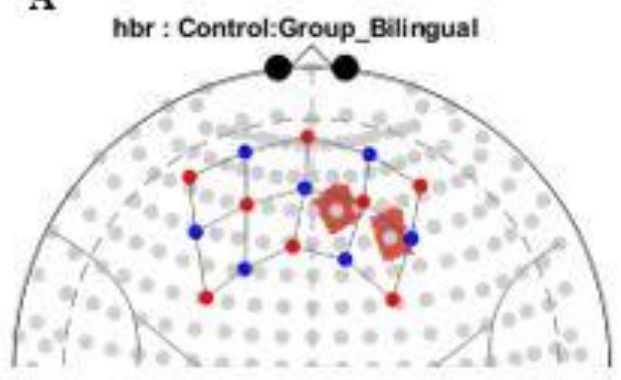

hbr : Color:Group_Bilingual

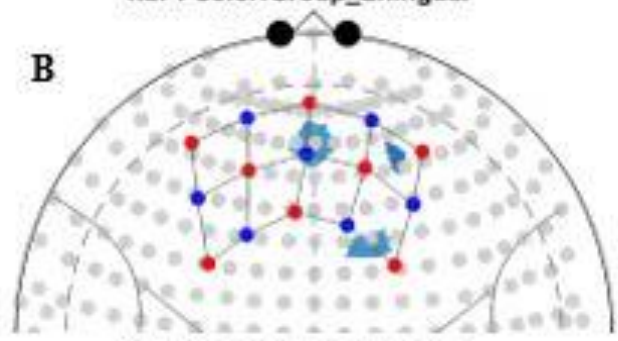

hbr : Shape:Group_Bilingual

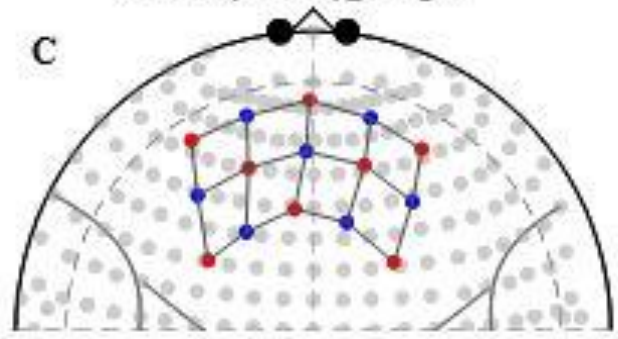

hbr : Border:Group_Belingual

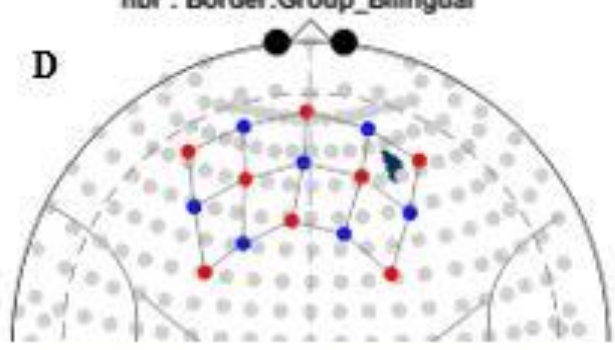

E

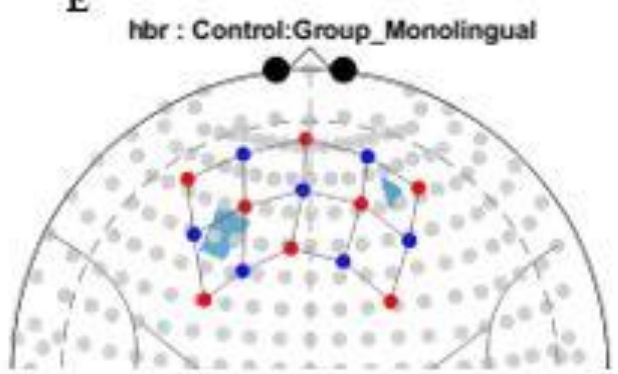

ther : Color:Group_Mondingual

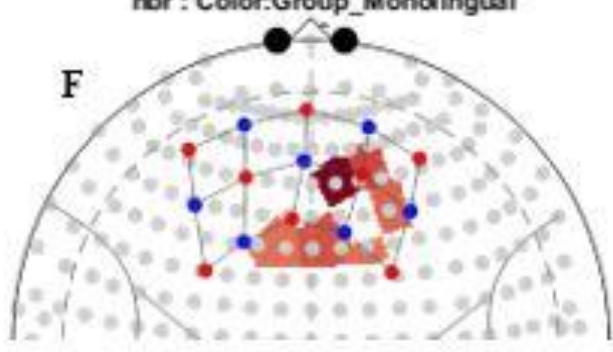

hbr : Shape:Group_Monolingual

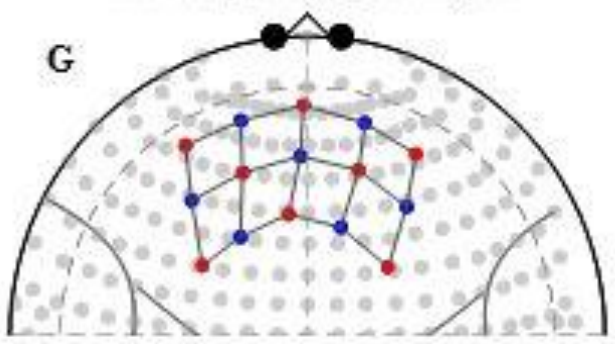

hbr : Border:Group_Monolingual

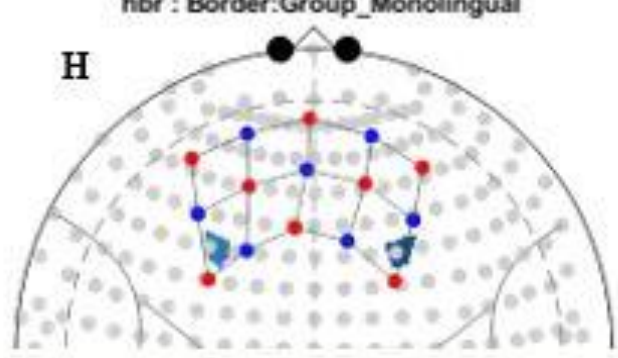

Figure 12: SPM T-map simulated via NIRS Toolbox. t-map of deoxy-hemoglobin averaged channels per language group.

The red areas represent positive t-values, which suggests that groups needed more $\mathrm{O}_{2}$ than the Hemodynamic Response Function (HRF) convolution predicted. The blue areas represent negative t-values, which suggests that the groups needed less $\mathrm{O}_{2}$ than the 
HRF convolution predicted. No similarities were noticed for any conditions. In sum, the bilinguals had statistically significant t-values in Channels 15 and 18 (Control), Channel 13, 19, 21 (Color) and Channel 21 (Border). Monolinguals had statistically significant differences in Channels 2 and 21 (Control), Channels 9, 10, 11, 15, 17, 18, 21 (Color), and Channels 1 and 20 (Border). The groups displayed differences in significant t-values (+/-) as well as location, during performance of those tasks. This implies that when the bilingual's activity increased the monolinguals activity decreased and vice versa per condition of the DCCS Task.

This then raised the question of why there were statistical differences among the group during the Control (blank card) trial. In theory, a statistical difference in hemoglobin level during the Control is problematic because the baselines must not have statistical differences to compare the groups. However, it is plausible that this data could also be telling. First, the First Control is different than the latter controls because the participants were not given a rest period to allow their neural activity to go back to their normal baselines. This meant that the data needed to be reanalyzed to account for the different baselines that were recorded.

\subsubsection{Analysis of Event Contrasts per Group}

In figure 13 below, the output of the SPM t-image per language group for oxyhemoglobin when the control (rest) condition is subtracted from the active conditions (i.e. Color, Shape, Border). 
A Color:Group_Bilingual-Control:Group_Bilingual

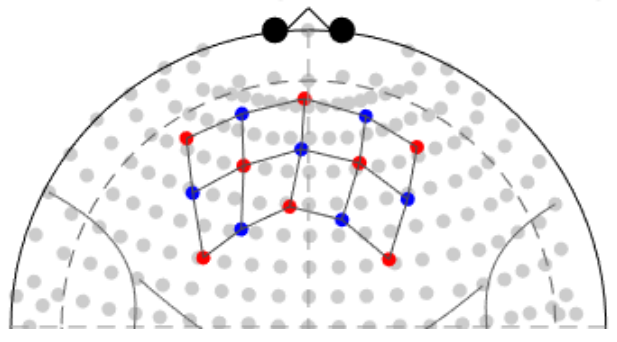

B

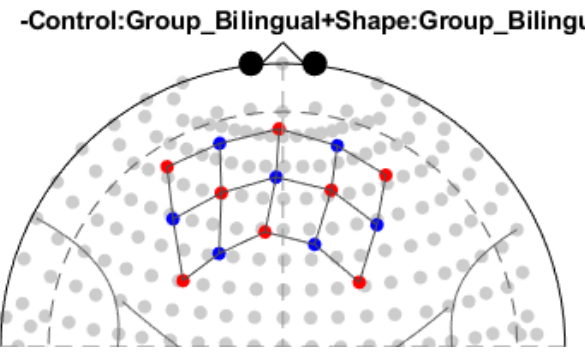

hbo : Border:Group_Bilingual-Control:Group_Bilingual

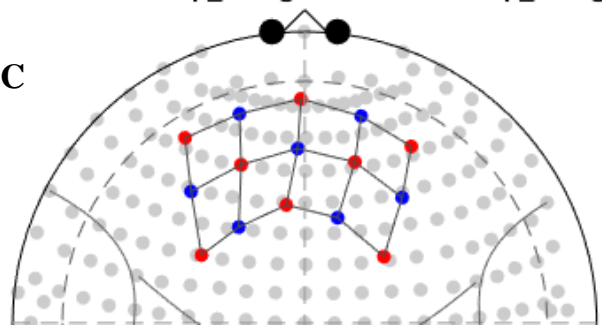

D

bo : Color:Group_Monolingual-Control:Group_Monolingual

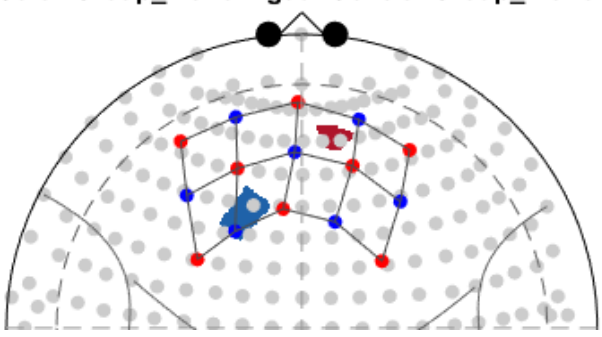

3

2

1

E bo :-Control:Group_Monolingual+Shape:Group_Monolingual

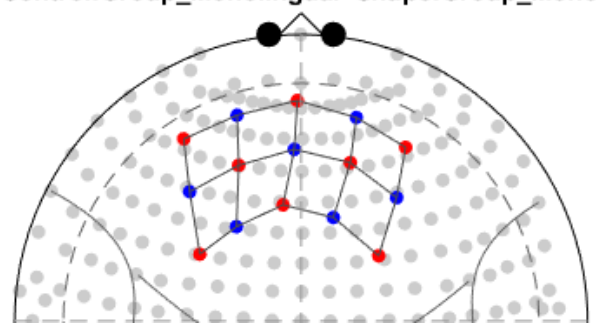

hbo : Border:Group_Monolingual-Control:Group_Monolingual

$\mathbf{F}$

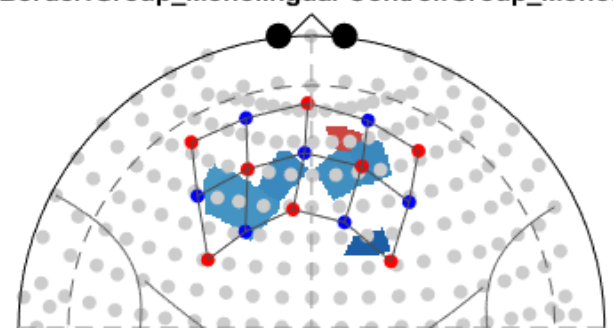

Figure 13: SPM T-map simulated via NIRS Toolbox. The oxy-hemoglobin contrasts for both language groups for the DCCS Task.

The red statistically significant areas exemplify positive t-values that remain when the control is subtracted from the active conditions. The blue statistically significant areas exemplify negative t-values that remain when the control is subtracted from the active conditions. Figure 13A-13C shows there is no statistically significant t-values for the bilinguals, which implies that there were no statistically significant variations from the GLM model compared to the HRF convolution per condition. As for the monolinguals, there are only statistically significant t-values for the Color and Border conditions. The red statistically significant areas correlate to Channel 17 for 13D and 13F. The blue statistically 
significant area in 13D corresponds with Channel 6. As for Figure 13F, the channels that correlate with the blue area are $5,6,8,15,17,19$.

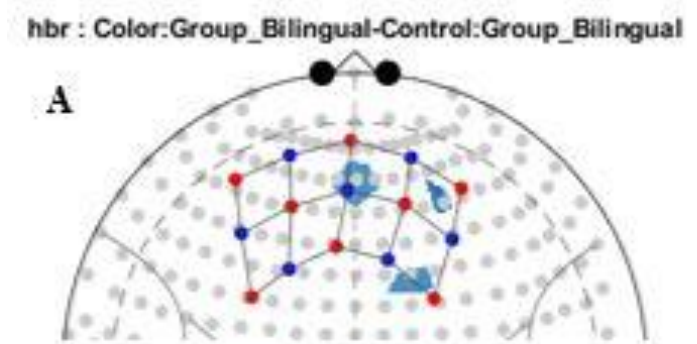

hbr:-Control:Group_Bilingual+Shape:Group_Bilingual

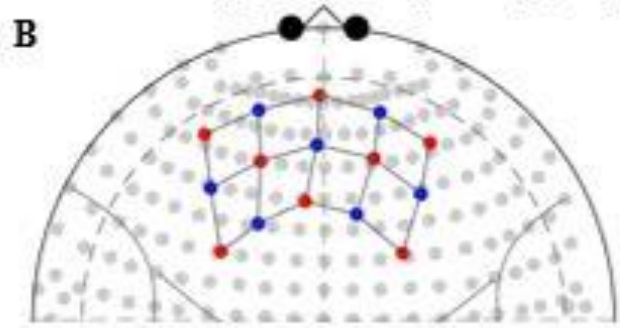

hbr : Border:Group_Bilingual_Control:Group_Bilingual

C

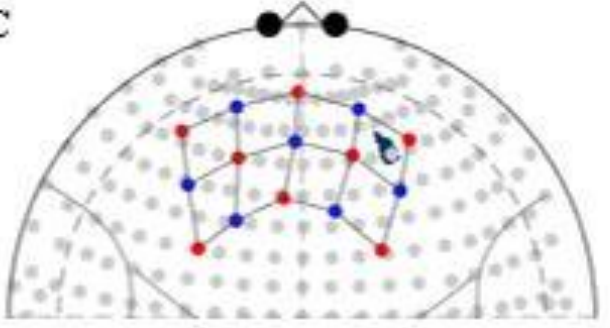

hbr : Color:Group_Mondingual-Control:Group_Monolingual

D

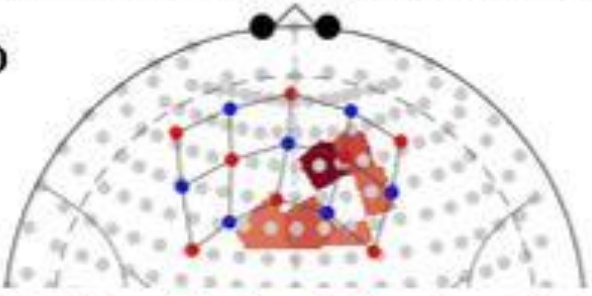

hbr :-Control:Group_Monolingual+Shape:Group_Monolingual

E

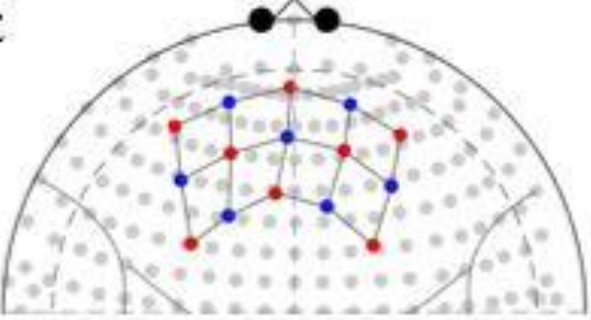

hbr : Border:Group_Monolingual-Control:Group_Monolingual

F

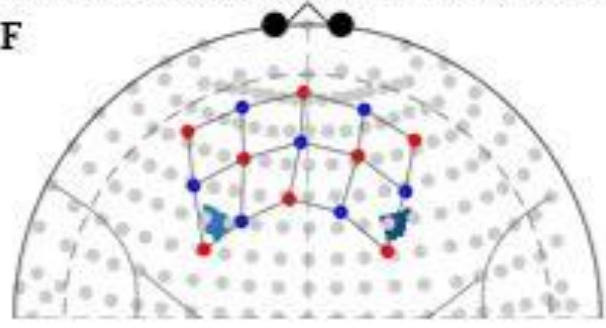

3

2

Figure 14: SPM T-map simulated via NIRS Toolbox. T-map of active - rest condition per language group for DCCS

The figure above shows the output of the SPM t-maps per language group for deoxy-hemoglobin when the control (rest) condition is subtracted from the active conditions. Figure 14A and 14C shows there is statistically significant t-values (blue) for the bilinguals, which means the Control values were larger than the active condition. There were no statistically significant t-values in the Shape conditions (14B and 14E), which implies that there were no statistically significant differences in the t-values for the Control and Shape conditions. As for the monolinguals, there is only statistically 
significant t-values for the Color and Border. Figure 14D and 14F shows there is statistically significant t-values, where red means the active condition t-values were larger than the control, and blue means the Control values were larger than the active condition. The reddest statistically significant areas correlate to Channel 15 in 14D. As for $14 \mathrm{~F}$, the areas that correlate to the blue areas are Channels 1, 2 and 20.

\subsection{2 fNIRS results for Go/No-Go task}

The SPM t-statistic outputs for a single participant and for each language group show the oxy- and deoxy-hemoglobin differences per condition and mixed conditions (contrasts). Some of the results were obtained by the making contrasts by subtracting the rest condition from the task condition (e.g. Shape-Control). The interpretation for both oxyhemoglobin and deoxy-hemoglobin are the same, but they have opposite meanings. To interpret these outputs, first the red and blue areas must be understood. For oxyhemoglobin, red areas signify an increase of oxygenated hemoglobin to those specific fNIRS channels, while blue represents a decrease. On the other hand, for deoxyhemoglobin, red areas signify an increase of deoxygenated hemoglobin to those specific fNIRS channels, while blue represents a decrease. It is important to note that blue areas do not represent deoxy-hemoglobin only.

\subsubsection{1 fNIRS Results for conditions of Go/No-Go task per Group}

Group data is shown below in Figure 15. In Figure 15, the SPM t-image per language group for each condition of the GNG Task is provided: 
A

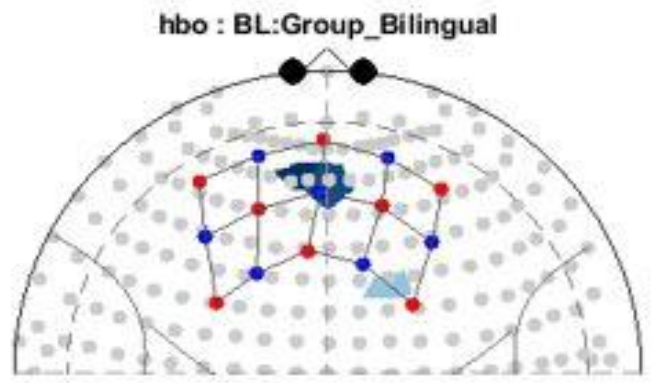

hbo: GO:Group_Bilingual

B

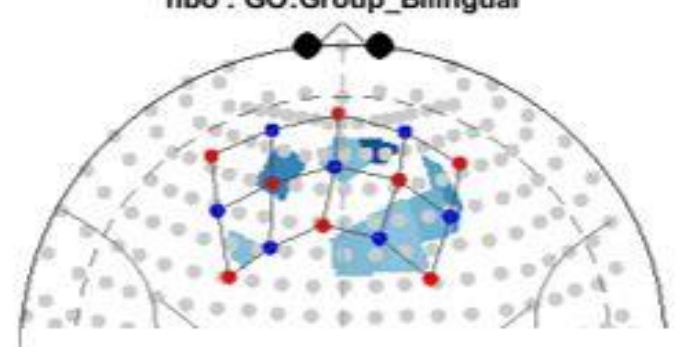

hbo : NG:Group_Bilingual

C

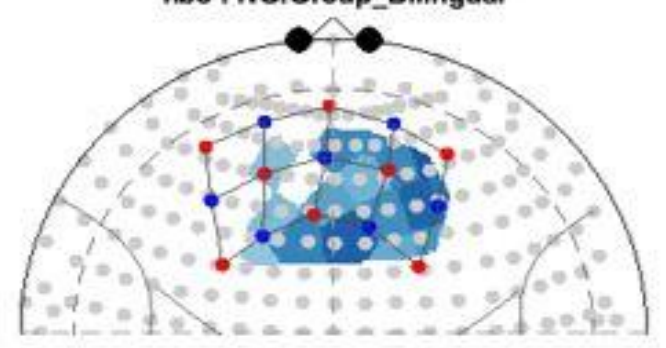

D

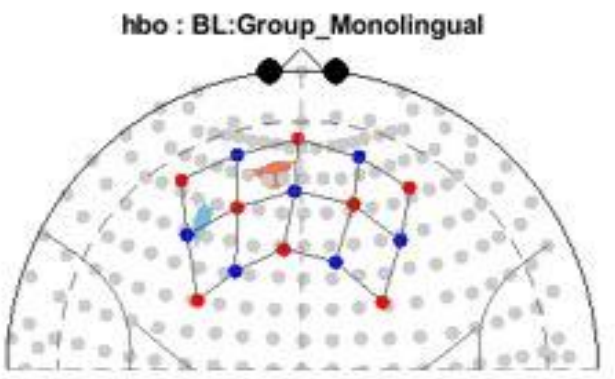

E

hbo : GO:Group_Monolingual

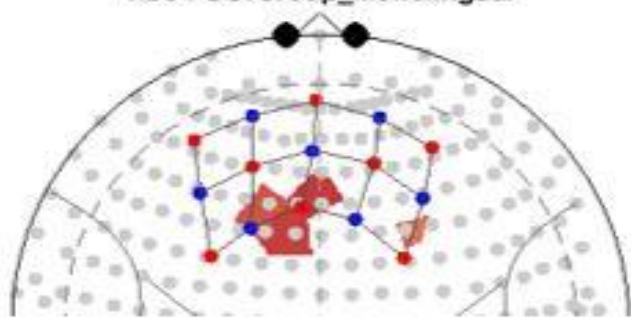

hbo : NG:Group_Monolingual

F

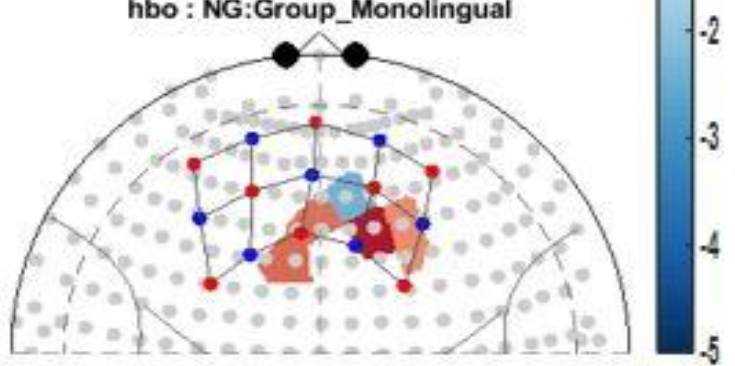

Figure 15: SPM T-map simulated via NIRS Toolbox. t-map of statistically significant areas in monolinguals vs bilinguals

Figure 15A - 15F show the statistically significant t-values for oxy-hemoglobin per language group for the each of the trials of the GNG task. For the control condition (15A and 15D), similarities in areas of statistically significance exist between groups for Channel 13. No similarities exist for the Go condition (15B and 15E), areas of statistically significance are the exact opposite with opposite t-values, which suggest that the bilinguals needed less $\mathrm{O}_{2}$ and from different areas of the brain. As for the No/Go condition $(\mathbf{1 5 C}$ and 15F), it contains the most similarities in statistically significant areas, which suggests that the bilinguals needed less $\mathrm{O}_{2}$ in these locations to perform the No/Go condition than the monolinguals. 
A

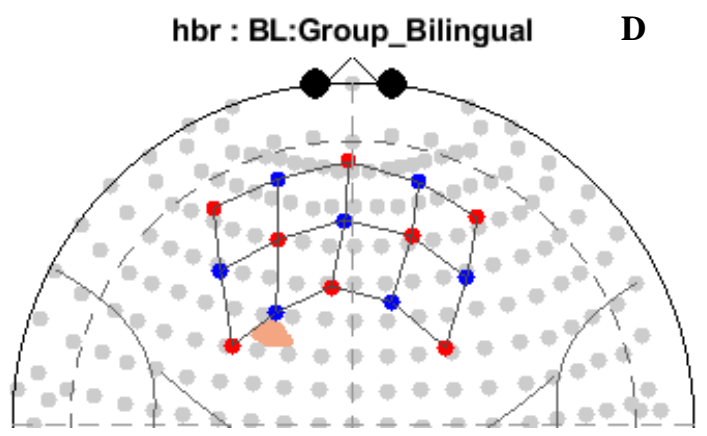

hbr : GO:Group_Bilingual

B

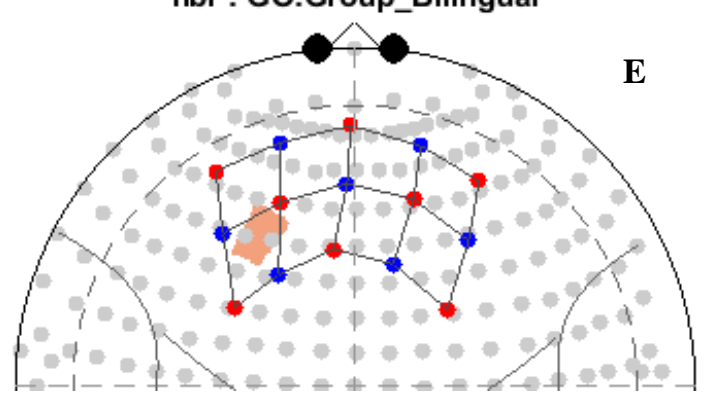

C

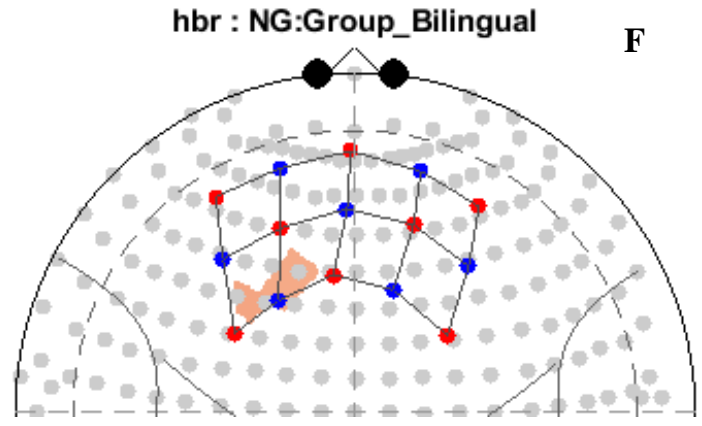

hbr : BL:Group_Monolingual

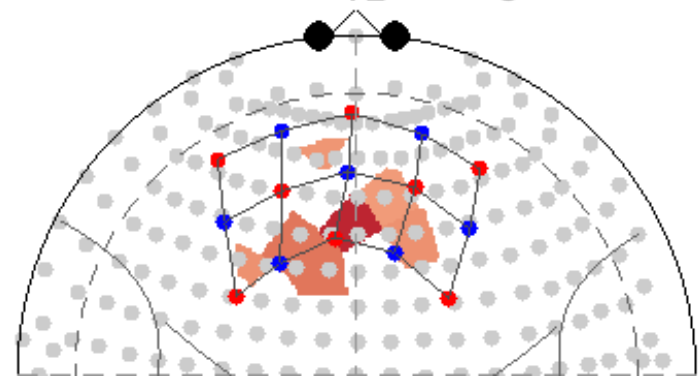

hbr : GO:Group_Monolingual

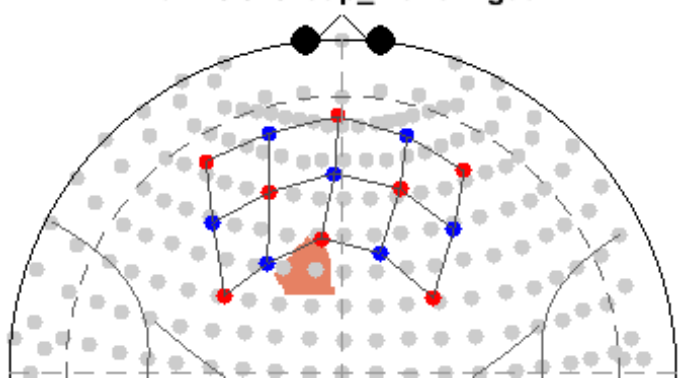

hbr : NG:Group_Monolingual

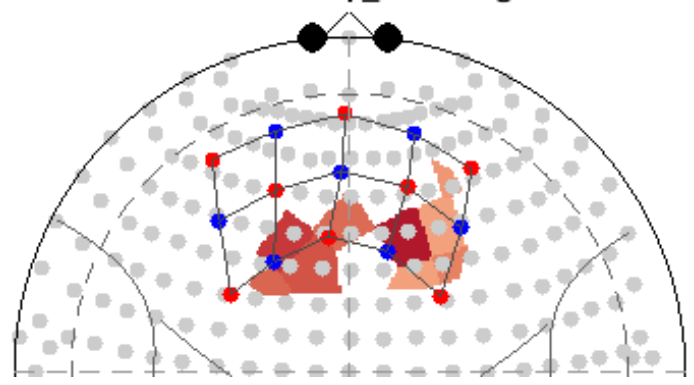

Figure 16: SPM T-map simulated via NIRS Toolbox. t-map of active - rest condition per language group for GNG with Deoxy-hemoglobin.

Figure 16A - 16F show the statistically significant t-values for deoxy-hemoglobin per language group for the each of the trials of the GNG task. For the control condition (16A and 16D), no similarities in areas of statistical significance exist between groups, where bilinguals show statistical significance in Channel 2, while monolinguals show statistical significance in Channel 10, 15, 16. For the Go condition (16B and 16E), no 
similarities exist between the groups, where bilinguals used Channel 5 and monolinguals use Channel 9. As for the $\mathrm{No} / \mathrm{Go}(\mathbf{1 6 C}$ and $16 \mathrm{~F})$, it contains one similarity in statistically significant areas (Channel 6) between the groups; however, the monolinguals showed more statistically significant areas, showing the difference in brain areas used for this task.

\subsubsection{Contrasts of Conditions for Go/No-Go for both hemoglobin states per Group}

Group data is shown below in Figure 17. In Figure 17, the SPM t-image contrasts per language group for each condition of the GNG Task is provided:

hbo :-GO:Group_Bilingual+NG:Group_Bilingual

A

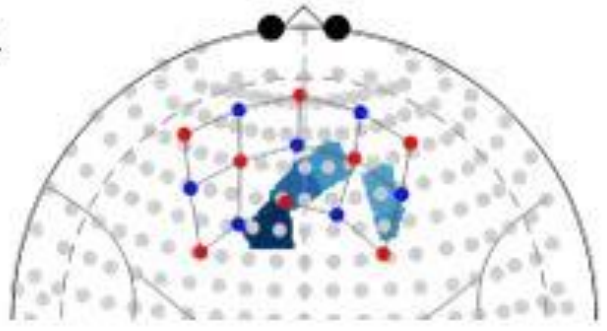

hbo :-GO:Group_Monolingual+NG:Group_Monolingual

B

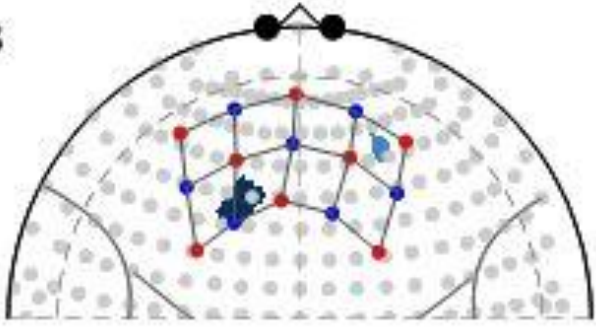

hbr:-GO:Group_Belingual+NG:Group_Bllingual

C

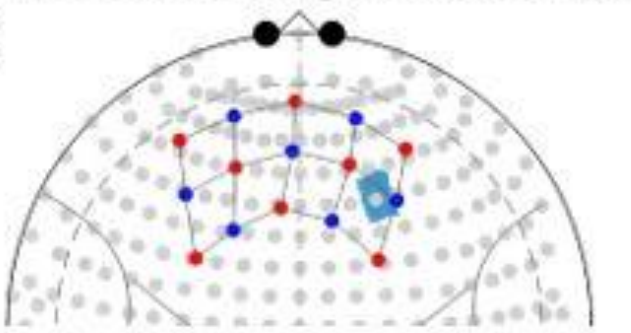

hbe :-GO:Group_Monolingual+NG:Group_Monolingual

D

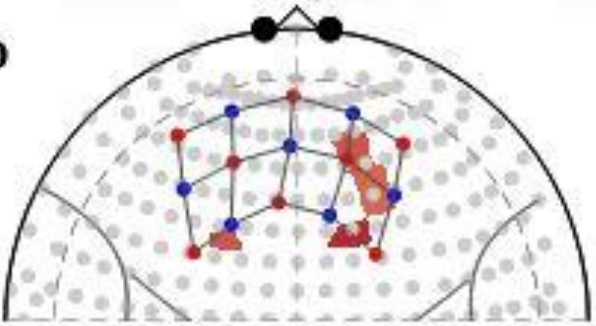

3

2

Figure 17: SPM T-map simulated via NIRS Toolbox. T-map of active - rest condition per language group for GNG for Oxy-hemoglobin.

Figure 17 shows the differences between the groups when comparing the two events, Go and No-Go. The Go trial stands as the control state, since the subject is just pressing the button for all stimulus. Therefore, to see any changes from the No-Go trial the base (i.e. Go trials) must be subtracted. 

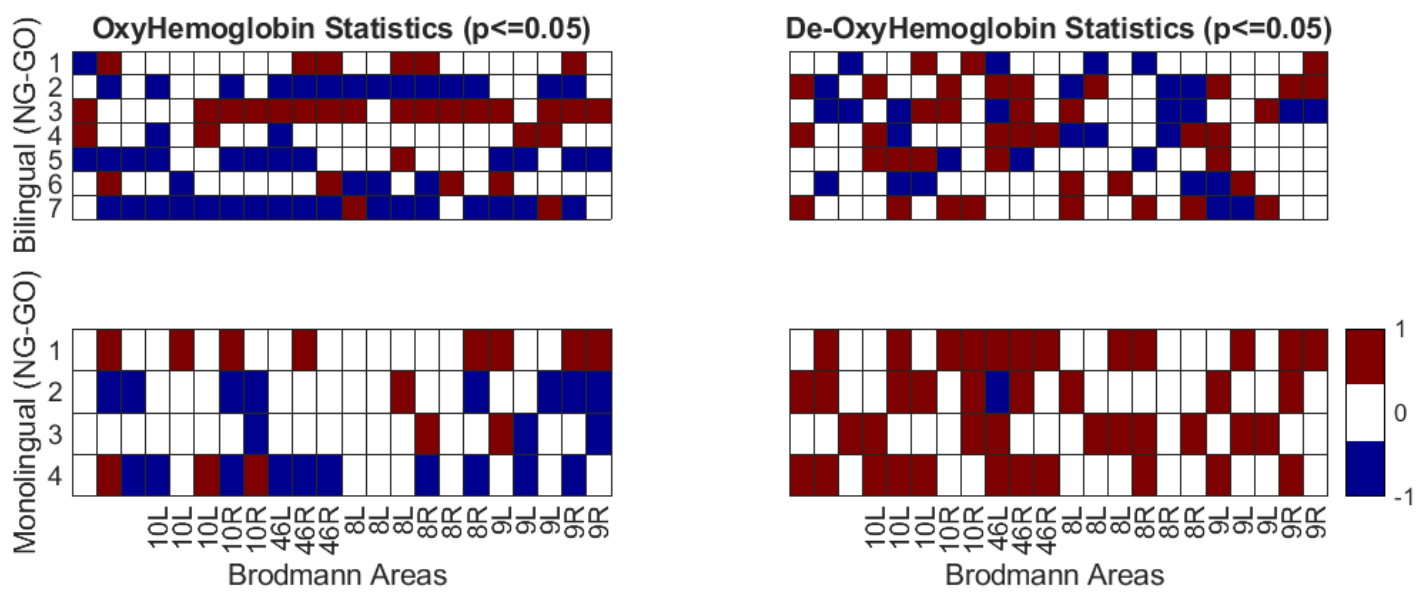

Figure 18: Significant beta values with Broadman areas obtained after calculating $N G$-values minus GO b-values.

Figure 18 gives a visualization of which Broadman areas may have been involved during for the Go-No/Go Task when the GO b-values are removed from the NG b-values per language group for both the oxy-hemoglobin and deoxy-hemoglobin states. I do not aim to make claims that these areas of the brain activated, as NIRS only allows us to claim with accuracy channel numbers. However, following the literature, it can be speculated that these Broadman areas may be implicated. Below these implications are drawn in summary.

\subsubsection{Summary for Research Question 2}

\subsubsection{DCCS}

Task activation was determined per condition and by subtracting the "Rest/Control Condition" from "Active Conditions". When evaluating the oxy-hemoglobin, it was determined that the red statistically significant areas indicated that the groups or individual required more $\mathrm{O}_{2}$ than the expected response accounted. The blue significant areas indicated that the groups or individual required less $\mathrm{O}_{2}$. Both groups showed similarities in statistically significant areas for the Control Conditi on - management of uncertainty (8R)and Border Condition - memory encoding and retrieval (10L) -, which were expected 
given the instructions. Results imply that the bilinguals also used regulation of self-control in the Control Condition, eye movement in the Color Condition, and regulation in the Shape Condition marked as red statistically significant areas, which were expected given the instructions. As for the Border Condition, this area was blue, which indicates that memory encoding and retrieval process did not require as much $\mathrm{O}_{2}$. The bilingual group generally required less oxygen to complete the DCCS Task compared to the monolingual group, who required more oxygen. For the monolinguals, results imply regulation was statistically significant during the Color Condition and memory encoding/retrieval in the Border Condition.

When evaluating the deoxy-hemoglobin, it was determined that the red statistically significant areas indicated that the groups or individual required less $\mathrm{O}_{2}$ than the expected response accounted, and the blue statistically significant areas indicated that less $\mathrm{O}_{2}$ was required. Both groups showed similarities in red statistically significant areas for the Color Condition - memory encoding and retrieval (10L) - and Shape Condition - use of language $(10 \mathrm{R})$ - which implies the groups did not need as much $\mathrm{O}_{2}$ for these conditions as expected. Results imply that the bilinguals also showed regulation (9R) and regulation of self-control (46R) in the Control Condition, regulation in the Shape Condition, and memory encoding/retrieval in the Border Condition marked as red significant areas, implies the groups did not need as much $\mathrm{O}_{2}$ for these conditions as expected. However, the Color Condition showed eye movement (8L) in blue, which indicates that the eye movement process did not require as much $\mathrm{O}_{2}$. This result coincides with the Hbo results summarized above because the oxy- and deoxy- $\mathrm{Hb}$ states are inversely related. For the monolinguals, results imply regulation and memory encoding/retrieval were red significant areas used in 
the Color Condition and use of language (10R) in the Shape Condition, which implies they did not require as much $\mathrm{O}_{2}$. As for the blue area in the Control Condition - regulation (9R) - this implies that the group required more $\mathrm{O}_{2}$ to complete the task.

\subsubsection{GNG}

Task activation was determined per condition and by subtracting the "Go" from "No-Go". The SPM t-images were interpreted in the same manner as the DCCS Task. When evaluating the Hbo SPM t-images, both groups had similarities in significant areas for the Blank Condition - use of language (10R) - and in the No/Go Condition - regulation (9R) and management of uncertainty (8R) - but had no similarities in the Go Condition. However, only (9R) for both groups were both blue, signaling less $\mathrm{O}_{2}$ was needed. Whereas, the other similar significant areas differ in $\mathrm{O}_{2}$ requirement. When evaluating the hbr SPM t-images, both groups had similarities in red significant areas for the No/Go Condition - eye movement $(8 \mathrm{~L})$ - only. The bilinguals showed red significant areas in $(8 \mathrm{~L})$ for the Blank Condition, (9L) for the Go Condition, and (8R) additionally for the No/Go Condition, while the monolinguals showed the (9L) for Blank Condition and (8L) for Go Condition. Results imply that less $\mathrm{O}_{2}$ was needed for both groups. No blue significant areas were observed for this $\mathrm{Hb}$ state. 


\section{CHAPTER 5: DISCUSSION AND CONCLUSION}

The results of this study show that bilingual preterm-born children are significantly faster $(\mathrm{U}=3.0, \mathrm{p}=.057)$ than preterm-born monolingual children in tasks measuring executive function (EF) and require less $O_{2}$ when recruiting oxygenated hemoglobin for EF. The study sought out to demonstrate the potential for enhancing preterm-born child's executive functioning skills through the daily use of two language, given that prematurity negatively impacts EF skills. The findings from this study coincide with the results of numerous studies (Fan, Liberman, Keysar, \& Kinzler, 2015; King \& Mackey, 2007; Kovács \& Mehler, 2009), showing that not only term-born, but also, preterm-born bilinguals individuals can reap the cognitive benefits of bilingualism. This bilingual advantage held even though the preterm-born bilingual children in our cohort were born smaller, earlier, and were in the NICU longer than the monolingual preterm-born children. This study is the first to show that the bilingual's significant activation areas (i.e. t-values) were average were lower than the monolinguals on both EF tasks, implying the bilingual group did not rely on as much $\mathrm{O}_{2}$ consumption as the monolingual group - and in a preterm-born population.

The DCCS Task, which measured cognitive flexibility and switching, provided quantitative data to determine if the pre-term bilinguals reaped the benefits of bilingualism like their term counter parts. The results showed that the pre-term bilingual children were about two times faster at providing responses, while still being just as accurate as the other group. This is a clear advantage, which explains the faster processing times. Although the two groups were placed in the same environment, the areas of neural recruitment used between groups to complete the tasks had some similarities but major differences per 
condition. The Go/No-Go Task, which measured inhibition, provided quantitative data to determine if the pre-term bilinguals' inhibition skills were better than the monolinguals. The GNG results were the same as the DCCS task; the bilingual preterm-born children were about two times faster than the monolinguals at providing responses, and at inhibiting responses.

From a psycholinguistic perspective, these data indicate that the daily use of two languages enhances EF. Monolingual children are not exposed to an environment in which they constantly must inhibit responses based on the task at hand. The bilinguals are exposed to different linguistic environments, and so must have the skill set to switch back and forth, inhibit the language not required for the task at hand, and know when to use which language determined by the social context. This resultantly improves EF in the brain. This may be why the bilingual children performed the two EF tasks significantly faster than the monolingual children, while having almost identical accuracy scores.

In terms of why the bilingual children required less $\mathrm{O}_{2}$, there are several theoretical postulations that may be implied. Initially, it was expected that the bilingual children would have more efficient neural recruitment of EF, and thus, more $\mathrm{O}_{2}$ levels. At least, this has been implied in the literature (e.g. Moriguchi \& Hiraki, 2013). It seems that so far, most of the literature has been working under the hypothesis that low hbO levels is congruent to poor cognition performance. The dataset here indicates the opposite. Maybe, it is the case that less oxygenated blood can be equivalented with more efficient EF recruitment. Theoretically, this makes sense: if the bilingual children are constantly having to switch back and forth between their two languages, their EF and its neural correlates are also more efficient, and so less effort expenditure is required. So far, one 
paper in the field seems to have found the same results. In a study by Causse, Chua, Peysakhovich, Del Campo, and Matton (2017), it was argued that the harder the task was, or in other words, the harder the mental effort, the less $O_{2}$ levels will be observed with the individuals who have the greatest mental efficiency. It may be the case that the daily use of two languages makes bilinguals more efficient in EF. It is possible that this is why the bilinguals were twice as fast and yet required statistically less $\mathrm{O}_{2}$ levels than the bilinguals.

\subsection{Implications for society}

There are about 15 million premature births in the USA alone, which is more than one of ten children ("Preterm birth," 2018). Those that survive are at a statistically higher risk to experience many mental and/or physical complications. Unfortunately, physicians who recommend that parents abandon their home language are not helping these children (Ward \& Beachy, 2003). The results from this study explicitly show that the bilingual children significantly performed better than their monolingual counterparts, thus disproving these oft-cited myths. Perhaps the most important outcome of this study is that the benefits of bilingualism repeatedly seen in term-born individuals can be extended to preterm-born children (Akhtar \& Menjivar, 2012; Bialystok et al., 2012). This has significant implications for society, because, if bilingualism can help premature-born children in EF, it can inform practice and health recommendations for families. Supporting the EF development of term- and of pre-term-born children is a critical mission for society because when children have strong EF schools, they do better in school and are set up to have better overall educational outcomes. Society is stronger when people are more educated, and resultantly, healthier. 
From a biomedical engineering standpoint, this study contributes to the methods currently being fine-tuned in the field on how to use fNIRS with young children. While the fNIRS technology is expanding, there is still not a consensus on how to reduce both physiological and motion artifact noise (among other factors). Two procedures were done for the present study, 4th order Butterworth filter was used as well as Temporal D Distributive Repair. I also reported on statistical levels observed per channel and drew plausible implications for where and how these might correlate to Broadmann's areas. Those results should be interpreted with caution however. With more procedures being shared, it is hoped that scholars can continue to collaborate and learn together on how to best utilize this technology given its benefits in working with young children.

From a clinical perspective, this study also contributes to knowledge about how we may help preterm-born children in the NICU. The most important finding would be to stop telling Hispanic or immigrant culturally and linguistically diverse parents to switch to English only with their child. In the Darcy Mahoney and Baralt (2016) cohort, every Hispanic parent reported being told by a pediatrician that they should switch to English only, given that their child was expected to have cognitive challenges due to his or her preterm birth. The study here provides the science that this practice is not evidencebased, and arguably, is unethical.

\subsection{Limitations of the study}

The most pressing limitation of this study was the small sample size. Recruiting righthanded children who were monolingual or balanced bilinguals (and with productive capacity), ages six to seven, and right-handed only, proved to be very challenging and took 
over a year. We also did not utilize a control group, for example term-born children, and doing so would have provided greater insight into the neural recruitment of EF in pretermborn children. We additionally experienced data loss with both the video and neural recordings, which further eliminated some of the participants. Although we were following Moriguchi and Hiraki's (2013) protocol, there are arguably also limitations associated with calculating reaction times by retrospectively watching videos; human error cannot be avoided here. Another major challenge was the way in which we classified the bilingual children. Many of the children who came to the lab, but whose data ultimately could not be used, had receptive knowledge of Spanish. It was purposeful to work with bilingual children with productive ability only; however, research is also needed on varying levels of proficiency to explore the bilingual edge. Some of the children with extreme cognitive deficits and/or cerebral palsy had to be excluded from the study as well due to inability to stay focused on tasks or too many involuntary movements, which resulted in poor fNIRS readings. Although fNIRS is less susceptible to movement, it was impossible to analyze their data because of excess noise, and the Temporal D Distributive Repair procedure was unable to amend this problem.

For future studies, it will be necessary to involve many more participants and with different age-ranges and perhaps both right- and left-handedness. More tasks that measure EF should also be used. It would also be insightful to explore different languages, and as mentioned above, children with varying degrees of proficiency in a second language. Finally, there are many different ways to approach NIRS data from a biomedical standpoint. Future studies could employ various methods to see if they yield different results. 


\subsection{Conclusion}

This study was the first to explore if the "bilingual advantage" exists in bilingual pre-term born children as compared to monolingual preterm-born children. The results reported on here show that the advantage does indeed exist. The bilingual preterm-born children were statistically faster than the monolingual children on both behavioral tasks measuring EF. There was also a faster exchange of oxy- and deoxy-hemoglobin in the bilingual group, meaning that their information processing was faster. Overall, the bilingual preterm-born children required statistically less $\mathrm{O}_{2}$ than the monolingual children. They were more efficient at neurally recruiting EF, and so required less hemoglobin. This provides the insight into how the neural recruitment of EF takes place in this population. The clinical implication of this study is that recommending that families with preterm-born children raise them as bilinguals may give their children them an edge despite their prematurity, and set them up to better develop the skills needed to perform well in school. 


\section{BIBLIOGRAPHY}

Akhtar, N., \& Menjivar, J. A. (2012). Cognitive and Linguistic Correlates of Early Exposure to More than One Language. Advances in Child Development and Behavior, 42, 41-78. https://doi.org/10.1016/B978-0-12-394388-0.00002-2

Als, H., Duffy, F. H., McAnulty, G. B., Rivkin, M. J., Vajapeyam, S., Mulkern, R. V., ... Eichenwald, E. C. (2004a). Early Experience Alters Brain Function and Structure. Pediatrics, 113(4 I), 846-857. https://doi.org/10.1542/peds.113.4.846

Als, H., Duffy, F. H., McAnulty, G. B., Rivkin, M. J., Vajapeyam, S., Mulkern, R. V, ... Eichenwald, E. C. (2004b). Early experience alters brain function and structure. Pediatrics, 113(4), 846-857. Retrieved from http://www.ncbi.nlm.nih.gov/pubmed/15060237

Barker, J. W., Aarabi, A., \& Huppert, T. J. (2013). Autoregressive model based algorithm for correcting motion and serially correlated errors in fNIRS. Biomedical Optics Express, 4(8), 1366-1379. https://doi.org/10.1364/BOE.4.001366

Bialystok, E., Barac, R., Blaye, A., \& Poulin-Dubois, D. (2010). Word mapping and executive functioning in young monolingual and bilingual children. Journal of Cognition and Development, 11(4), 485-508. https://doi.org/10.1080/15248372.2010.516420

Bialystok, E., Craik, F. I. M., \& Luk, G. (2012, April 1). Bilingualism: Consequences for mind and brain. Trends in Cognitive Sciences. Elsevier Current Trends. https://doi.org/10.1016/j.tics.2012.03.001

Bialystok, E., \& Shapero, D. (2005). Ambiguous benefits: The effect of bilingualism on reversing ambiguous figures. Developmental Science, 8(6), 595-604. https://doi.org/10.1111/j.1467-7687.2005.00451.x

Biran, V., Verney, C., \& Ferriero, D. M. (2012). Perinatal cerebellar injury in human and animal models. Neurology Research International. https://doi.org/10.1155/2012/858929

Brito, N., \& Barr, R. (2012). Influence of bilingualism on memory generalization during infancy. Developmental Science, 15(6), 812-816. https://doi.org/10.1111/j.14677687.2012.1184.x

Buchweitz, A., \& Prat, C. (2013). The bilingual brain: Flexibility and control in the human cortex. Physics of Life Reviews, 10, 428-443. https://doi.org/10.1016/j.plrev.2013.07.020

Buss, A. T., Fox, N., Boas, D. A., \& Spencer, J. P. (n.d.). Probing the early development of visual working memory capacity with functional near-infrared spectroscopy. NeuroImage.

Comishen, K. J., Bialystok, E., \& Adler, S. A. (2019). The Impact of Bilingual Environments on Selective Attention in Infancy. Developmental Science, e12797. https://doi.org/10.1111/desc. 12797

Cunha, A. B., Babik, I., Ross, S. M., Logan, S. W., Galloway, J. C., Clary, E., \& Lobo, M. A. (2018). Prematurity may negatively impact means-end problem solving across the first two years of life. Research in Developmental Disabilities, 81, 24-36. https://doi.org/10.1016/J.RIDD.2018.03.007

Darcy Mahoney, A., \& Baralt, M. (2016). Bilingualism and executive inhibitory control 
in 4- and 5-year-old preterm born children: A pilot study. National Association of Neonatal Nurses Research, 16.

Dimes, M. of. (2017). 2017 Premature Birth Report Card. Marchofdimes.Org. Retrieved from https://www.marchofdimes.org/peristats/tools/ReportFiles/PrematureBirth/2017/rc/p df/PrematureBirthReportCard-UnitedStates-2017.pdf

Doebel, S., \& Zelazo, P. D. (2015). A meta-analysis of the Dimensional Change Card Sort: Implications for developmental theories and the measurement of executive function in children. Developmental Review, 38, 241-268. https://doi.org/10.1016/J.DR.2015.09.001

Edgin, J. O., Inder, T. E., Anderson, P. J., Hood, K. M., Clark, C. a C., \& Woodward, L. J. (2008). Executive functioning in preschool children born very preterm: relationship with early white matter pathology. Journal of the International Neuropsychological Society : JINS, 14(1), 90-101. https://doi.org/10.1017/S1355617708080053

Eichenwald, E. C., \& Stark, A. R. (2008). Management and outcomes of very low birth weight. The New England Journal of Medicine. https://doi.org/10.1056/NEJMra0707601

Fan, S. P., Liberman, Z., Keysar, B., \& Kinzler, K. D. (2015). The Exposure Advantage. Psychological Science, 26(7), 1090-1097. https://doi.org/10.1177/0956797615574699

Fishburn, F. A., Hlutkowsky, C. O., Bemis, L. M., Huppert, T. J., Wakschlag, L. S., \& Perlman, S. B. (2019). Irritability uniquely predicts prefrontal cortex activation during preschool inhibitory control among all temperament domains: A LASSO approach. NeuroImage, 184, 68-77. https://doi.org/10.1016/j.neuroimage.2018.09.023

Gonzalez-Barrero, A. M., \& Nadig, A. S. (2017). Can Bilingualism Mitigate Set-Shifting Difficulties in Children With Autism Spectrum Disorders? Child Development. https://doi.org/10.1111/cdev.12979

Hackman, D. A., Gallop, R., Evans, G. W., \& Farah, M. J. (2015). Socioeconomic status and executive function: Developmental trajectories and mediation. Developmental Science, 18(5), 686-702. https://doi.org/10.1111/desc.12246

Head, L. M., Baralt, M., \& Darcy-Mahoney, A. E. (2014). Bilingualism as a potential strategy to improve executive function in preterm infants.pdf. Atlanta, Georgia. https://doi.org/http://dx.doi.org/10.1016/j.pedhc.2014.08.015

Jacques, S. L. (2013). Optical properties of biological tissues: a review. Physics in Medicine and Biology, 58(11), R37-R61. https://doi.org/10.1088/00319155/58/11/R37

Katsuki, F., \& Constantinidis, C. (2014). Bottom-Up and Top-Down Attention. The Neuroscientist, 20(5), 509-521. https://doi.org/10.1177/1073858413514136

King, K., \& Mackey, A. (2007). The bilingual edge: why, when, and how to teach your child a second language. In International Journal of Bilingual Education and Bilingualism (pp. 8-11). https://doi.org/10.1080/13670050802645942

Kocsis, L., Herman, P., \& Eke, A. (2006). The modified Beer-Lambert law revisited. Physics in Medicine and Biology, 51(5), N91-N98. https://doi.org/10.1088/0031$9155 / 51 / 5 / \mathrm{N} 02$ 
Kolb, B., \& Gibb, R. (2011). Brain Plasticity and Behaviour in the Developing Brain. Journal of the Canadian Academy of Child and Adolescent Psychiatry, 20(4), 265276. https://doi.org/10.1016/B978-0-444-63327-9.00005-9

Kopton, I. M., \& Kenning, P. (2014). Near-infrared spectroscopy (NIRS) as a new tool for neuroeconomic research. Frontiers in Human Neuroscience, 8(August), 1-13. https://doi.org/10.3389/fnhum.2014.00549

Kovacs, A. M., \& Mehler, J. (2009). Cognitive gains in 7-month-old bilingual infants. Proceedings of the National Academy of Sciences. https://doi.org/10.1073/pnas.0811323106

Kovács, A. M., \& Mehler, J. (2009). Cognitive gains in 7-month-old bilingual infants. Proceedings of the National Academy of Sciences of the United States of America, 106(16), 6556-6560. https://doi.org/10.1073/pnas.0811323106

Kroll, J., Karolis, V., Brittain, P. J., Tseng, C.-E. J., Froudist-Walsh, S., Murray, R. M., \& Nosarti, C. (2017). Real-Life Impact of Executive Function Impairments in Adults Who Were Born Very Preterm. Journal of the International Neuropsychological Society, 23(05), 381-389. https://doi.org/10.1017/s1355617717000169

Limperopoulos, C., Benson, C. B., Bassan, H., Disalvo, D. N., Kinnamon, D. D., Moore, M., ... du Plessis, A. J. (2005). Cerebellar hemorrhage in the preterm infant: ultrasonographic findings and risk factors. Pediatrics, 116(3), 717-724. https://doi.org/10.1542/peds.2005-0556

Low, L. K., \& Cheng, H. J. (2006). Axon pruning: An essential step underlying the developmental plasticity of neuronal connections. Philosophical Transactions of the Royal Society B: Biological Sciences, 361(1473), 1531-1544. https://doi.org/10.1098/rstb.2006.1883

Marton, K., Kelmenson, L., \& Pinkhasova, M. (2007). Inhibition control and working memory capacity in children with SLI. Psikhologyah : Ketav 'et Mada'i Yisre'eli Le-'iyun Ule-Mehkar, 50(2), 110-121. https://doi.org/10.2117/psysoc.2007.110

McCarty, M. E., Clifton, R. K., \& Collard, R. R. (1999). Problem solving in infancy: the emergence of an action plan. Developmental Psychology, 35(4), 1091-1101. https://doi.org/10.1037/0012-1649.35.4.1091

Moriguchi, Y., \& Hiraki, K. (2013). Prefrontal cortex and executive function in young children: a review of NIRS studies. Frontiers in Human Neuroscience, 7(December), 1-9. https://doi.org/10.3389/fnhum.2013.00867

Nosarti, C., Giouroukou, E., Micali, N., Rifkin, L., Morris, R. G., \& Murray, R. M. (2007). Impaired executive functioning in young adults born very preterm. Journal of the International Neuropsychological Society, 13(4), 571-581. https://doi.org/10.1017/S1355617707070725

Orchinik, L. J. (2014). EFFECTS OF EXTREME PREMATURITY ON DOMAINS OF EXECUTIVE FUNCTION, (December).

Park, S. (2014). Bilingualism and Children with Autism Spectrum Disorders : Issues , Research , and Implications. Nys Tesol Journal, 1(2), 122-129. Retrieved from http://journal.nystesol.org/july2014/122park.pdf

Poulin-Dubois, D., Blaye, A., Coutya, J., \& Bialystok, E. (2011). The effects of bilingualism on toddlers' executive functioning. J Exp Child Psychol, 108(3), 567579. https://doi.org/10.1038/nbt.3121.ChIP-nexus

Preterm birth. (2018). Retrieved March 20, 2019, from https://www.who.int/news- 
room/fact-sheets/detail/preterm-birth

Prior, A., \& Macwhinney, B. (2010). A bilingual advantage in task switching. Bilingualism, 13(2), 253-262. https://doi.org/10.1017/S1366728909990526

Rivkin, M. J., Wolraich, D., Als, H., McAnulty, G., Butler, S., Conneman, N., ... Mulkern, R. V. (2004). Prolonged T $2 *$ values in newborn versus adult brain:

Implications for fMRI studies of newborns. Magnetic Resonance in Medicine, 51(6), 1287-1291. https://doi.org/10.1002/mrm.20098

Rose, S. A., Feldman, J. F., \& Jankowski, J. J. (2009). Information processing in toddlers: Continuity from infancy and persistence of preterm deficits. Intelligence, 37(3), 311-320. https://doi.org/10.1016/j.intell.2009.02.002

Rysavy, M. A., \& Ehret, D. E. Y. (2019, March 26). Extremely Preterm Birth Outcomes in Sweden. JAMA - Journal of the American Medical Association, 321(12), 11631164. https://doi.org/10.1001/jama.2019.2020

Szaflarski, J. P., Rajagopal, A., Altaye, M., Byars, A. W., Jacola, L., Schmithorst, V. J., ... Holland, S. K. (2012). Left-handedness and language lateralization in children. Brain Research, 1433, 85-97. https://doi.org/10.1016/j.brainres.2011.11.026

Taylor, H. G., \& Clark, C. A. C. (2016). Executive function in children born preterm: Risk factors and implications for outcome. Seminars in Perinatology, 40(8), 520529. https://doi.org/10.1053/j.semperi.2016.09.004

Torres, J. (2013). Reseña de: Montrul, Silvina. (2013). El bilingüismo en el mundo hispanohablante. Malden; Oxford: Wiley-Blackwell. 330 p. ISBN 978-0-470-657218. Revista de Humanidades. https://doi.org/10.5944/rdh.20.2013.12907

Wang, J., Zhang, J., Zheng, H., Li, J., Liu, D., Li, H., ... Wong, G. K.-S. (2004). Mouse transcriptome: Neutral evolution of 'non-coding' complementary DNAs. Nature, 431(7010), 3017. https://doi.org/10.1038/nature03016

Ward, R. M., \& Beachy, J. C. (2003). Neonatal complications following preterm birth. BJOG: An International Journal of Obstetrics \& Gynaecology, 110, 8-16. https://doi.org/10.1046/j.1471-0528.2003.00012.x 